\title{
The Hydroxyproline Proteome of HeLa cells with Emphasis on the Active Sites of Protein Disulfide Isomerases.
}

\section{Bruce C. Onisko ${ }^{1^{*}}$}

${ }^{1}$ OniPro LLC, Berkeley, CA, USA 94707

*Corresponding author. E-mail: bconisko@gmail.com

Table of Contents:

Page S-1. Cover Sheet:

Page S-2 to S-40. Supplemental Figures S1 to S38. MS-MS spectra of the hydroxyproline peptides identified in this study. Figure Legends include the amino acid sequence of the modified peptide as detailed in Supplemental Table S1.

Supplemental Table S1: Hydroxyproline peptides identified in this study. Excel spreadsheet is a separate file. 
Raw File

Scan Method Score $\mathrm{m} / \mathrm{z}$ Gene names

20150708_OE3_UPLC8_DBJ_OC_HELA_39frac_GluC_34 32012 FTMS; HCD 179.46969 .43 PDIA6

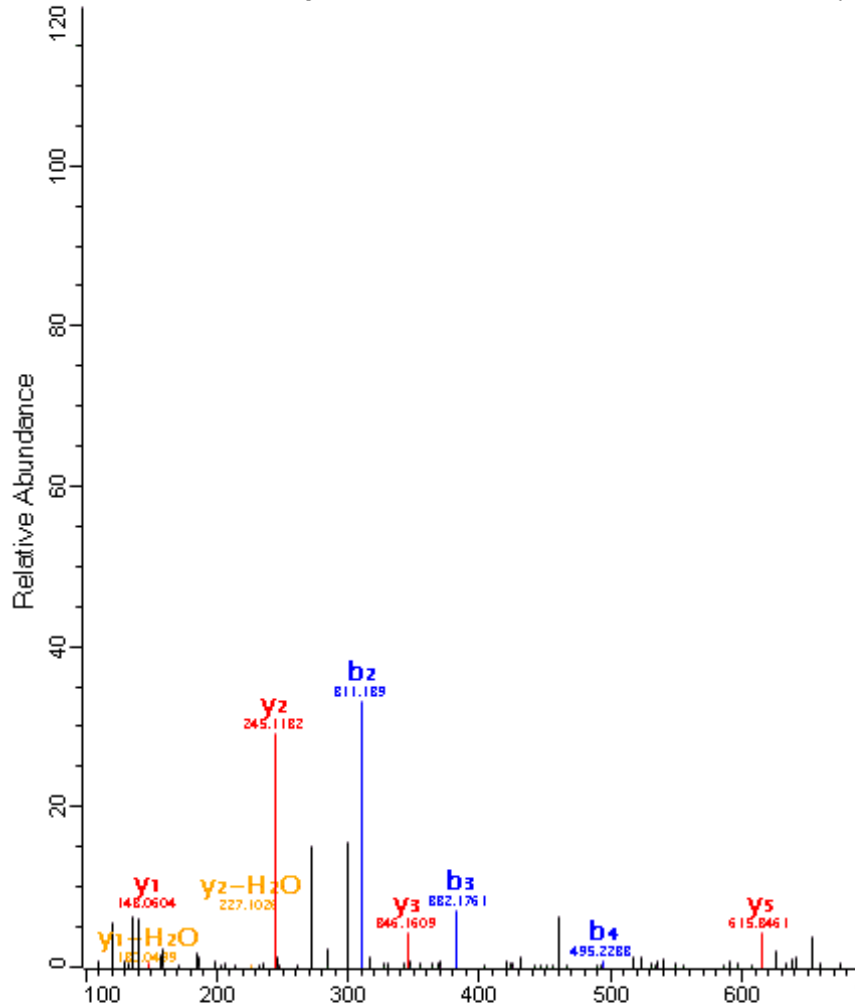

Sequence PSMs Fragments Properties

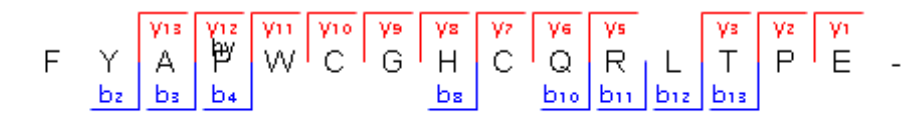

Figure S1. FYAP[Hydroxylation on P]WCGHCQRLTPE.

Page S-2 
\begin{tabular}{llllll} 
Raw File & \multicolumn{1}{l}{ Scan } & Method & Score & $\mathbf{m} / \mathbf{z}$ & Gene names \\
20150708 OES_UPLC8_DBJ_OC_HELA_39frac_CluC_33 & 28675 & FTMS; HCD & 99.45 & 725 & PDIA4
\end{tabular}

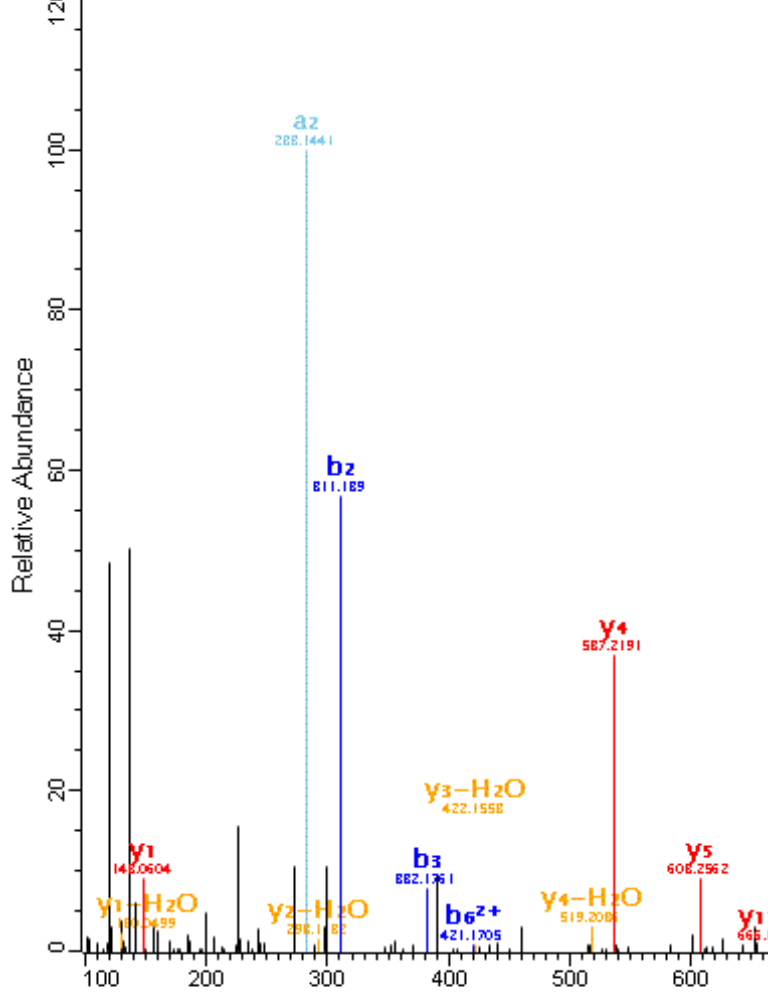
600

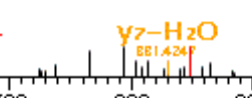

Sequence PSMs Fragments Properties

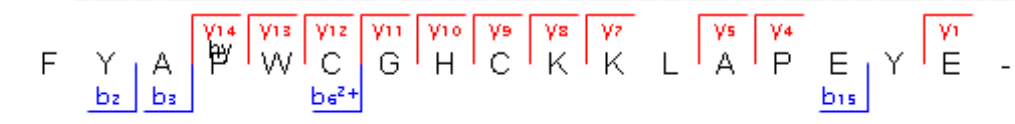

Figure S2. FYAP[Hydroxylation on P]WCGHCKKLAPEYE.

Page S-3 


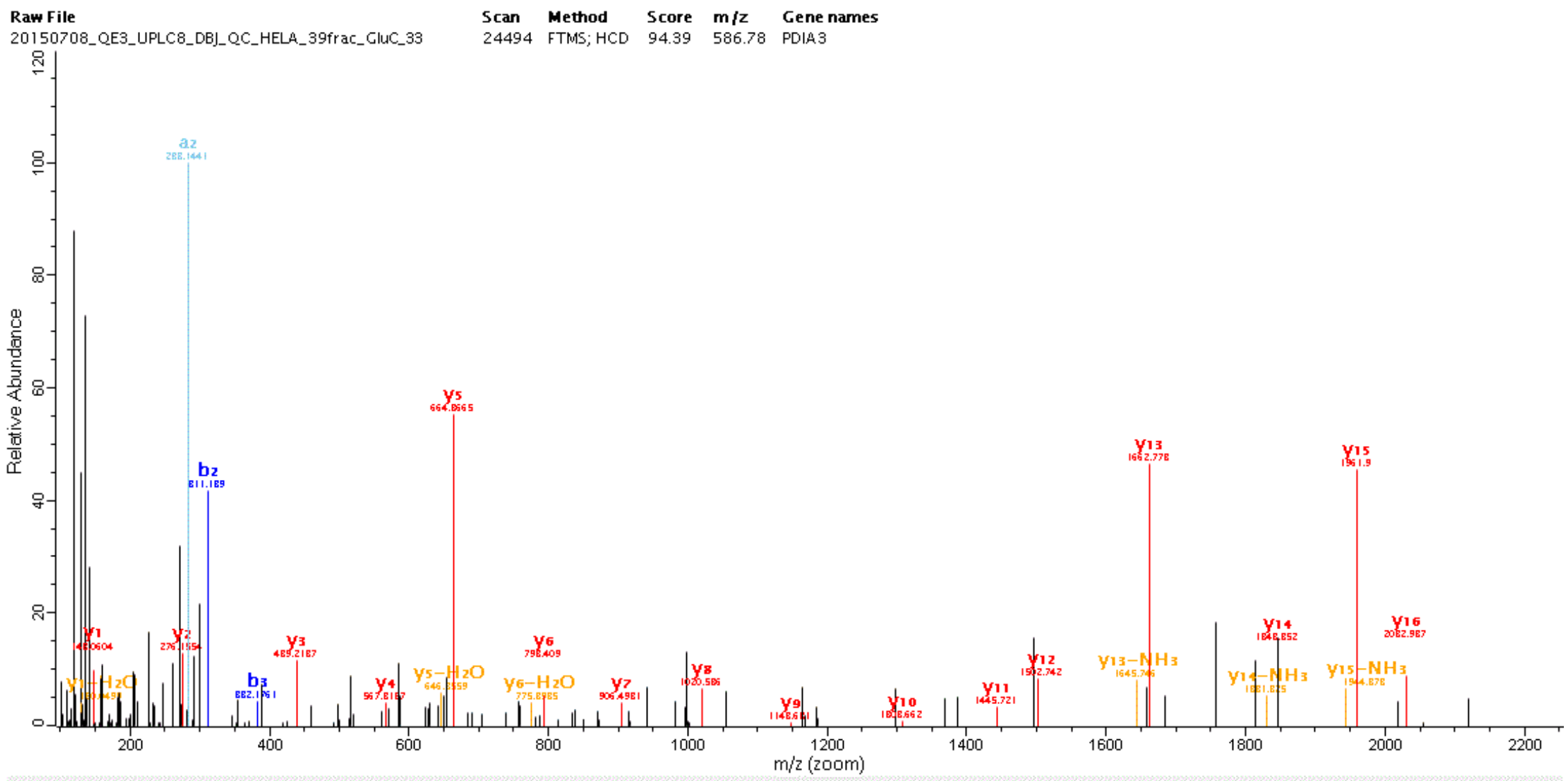

Sequence PSMs Fragments Properties

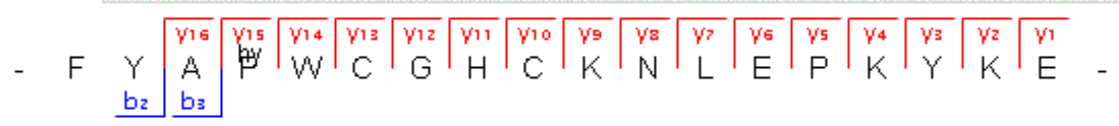

Figure S3. FYAP[Hydroxylation on P]WCGHCKNLEPKYKE. 
Raw File

20150708 QEE3_UPLC8_DBj_QC_HELA_39frac_CluC_15

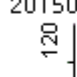

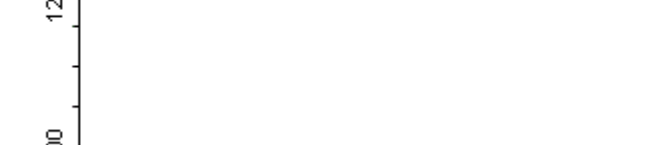

문
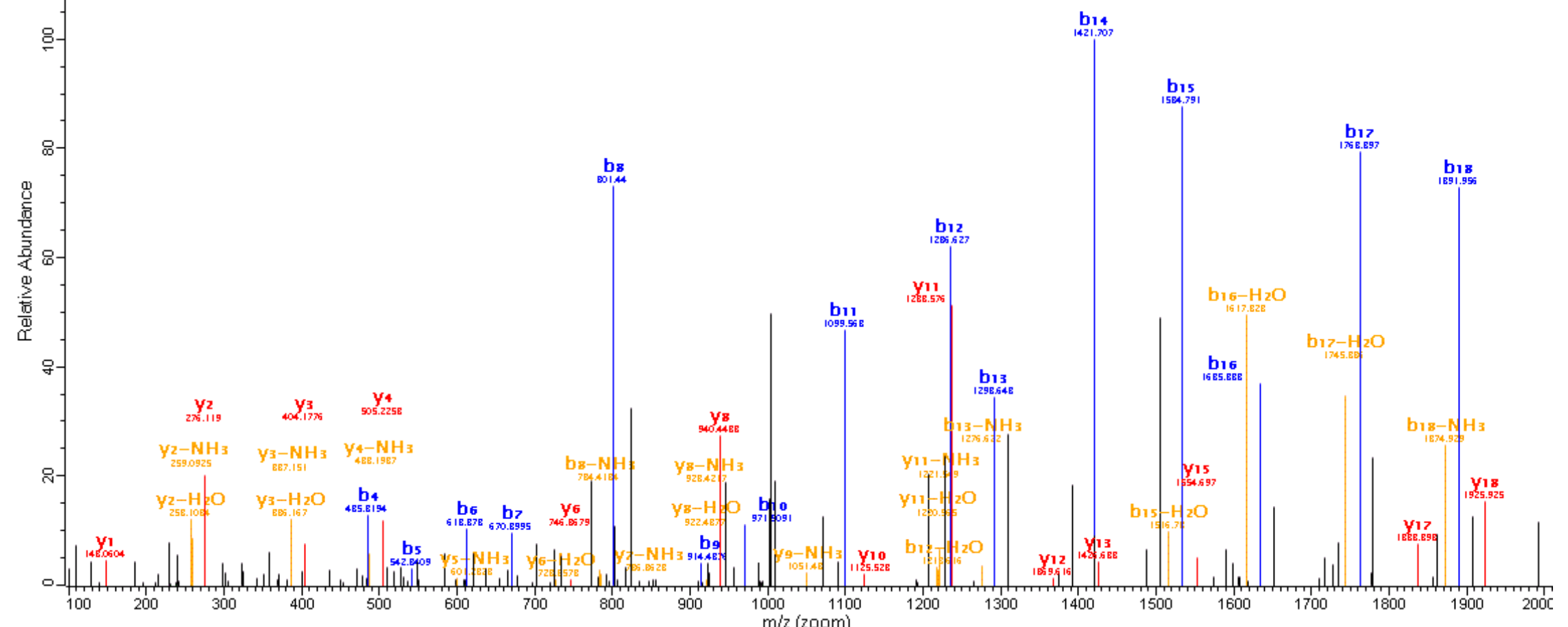

Sequence PSMs Fragments Properties

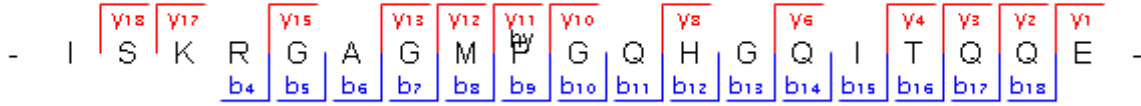

Figure S5. ISKRGAGMP[Hydroxylation on P]GQHGQITQQE. 


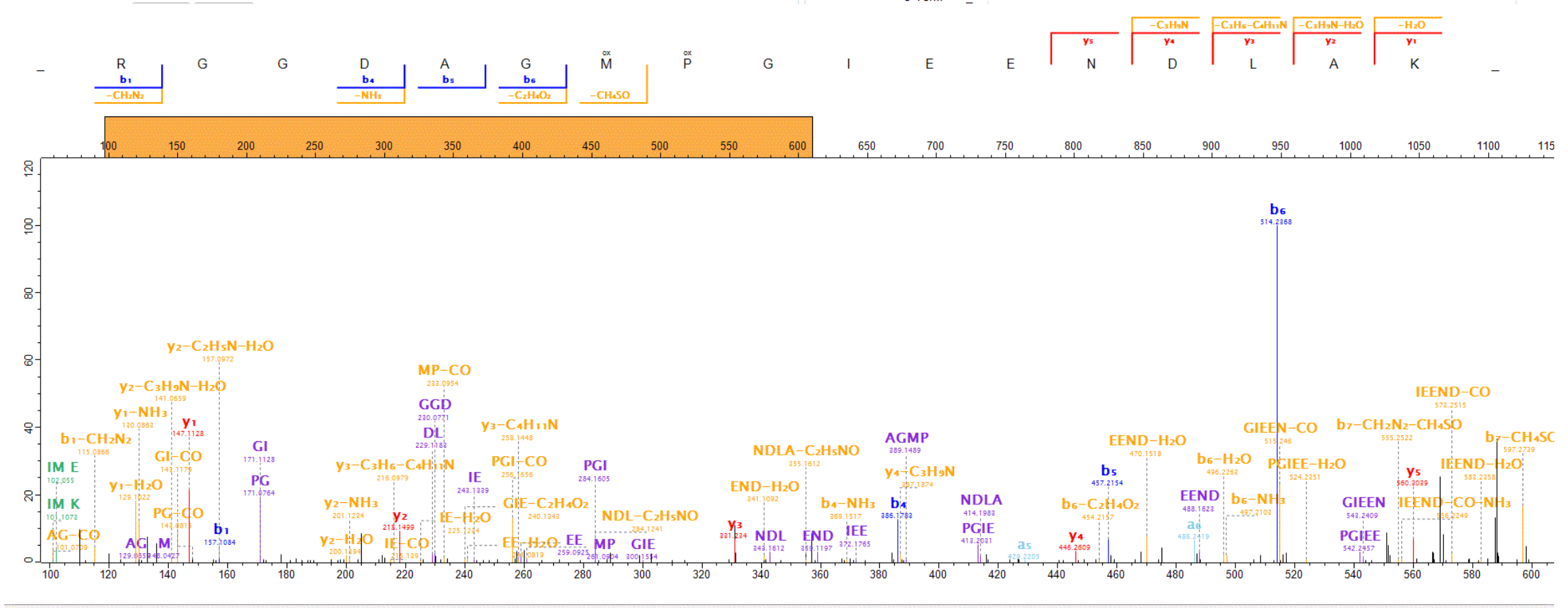

Figure S6a. RGGDAGM[Oxidation on M]P[Hydroxylation on P]GIEENDLAK_m/z_100-600. 


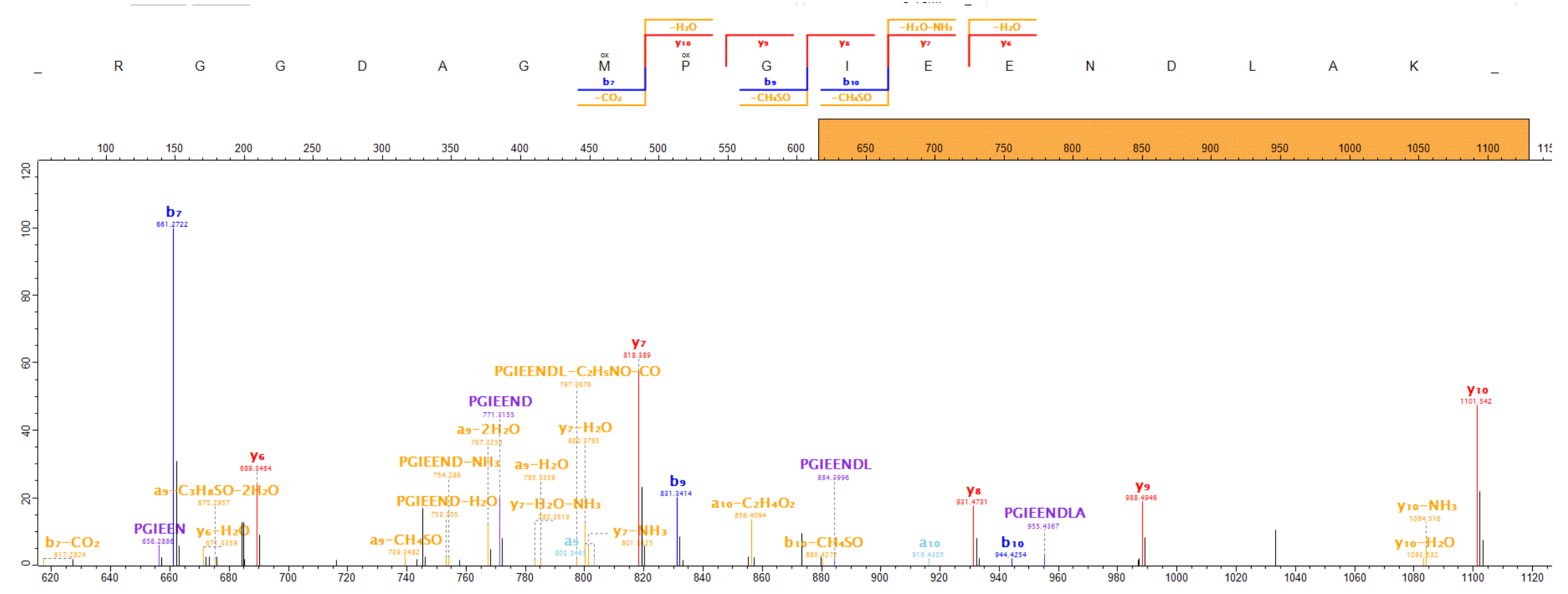

Figure S6b. RGGDAGM[Oxidation on M]P[Hydroxylation on P]GIEENDLAK_m/z_620-1120. 


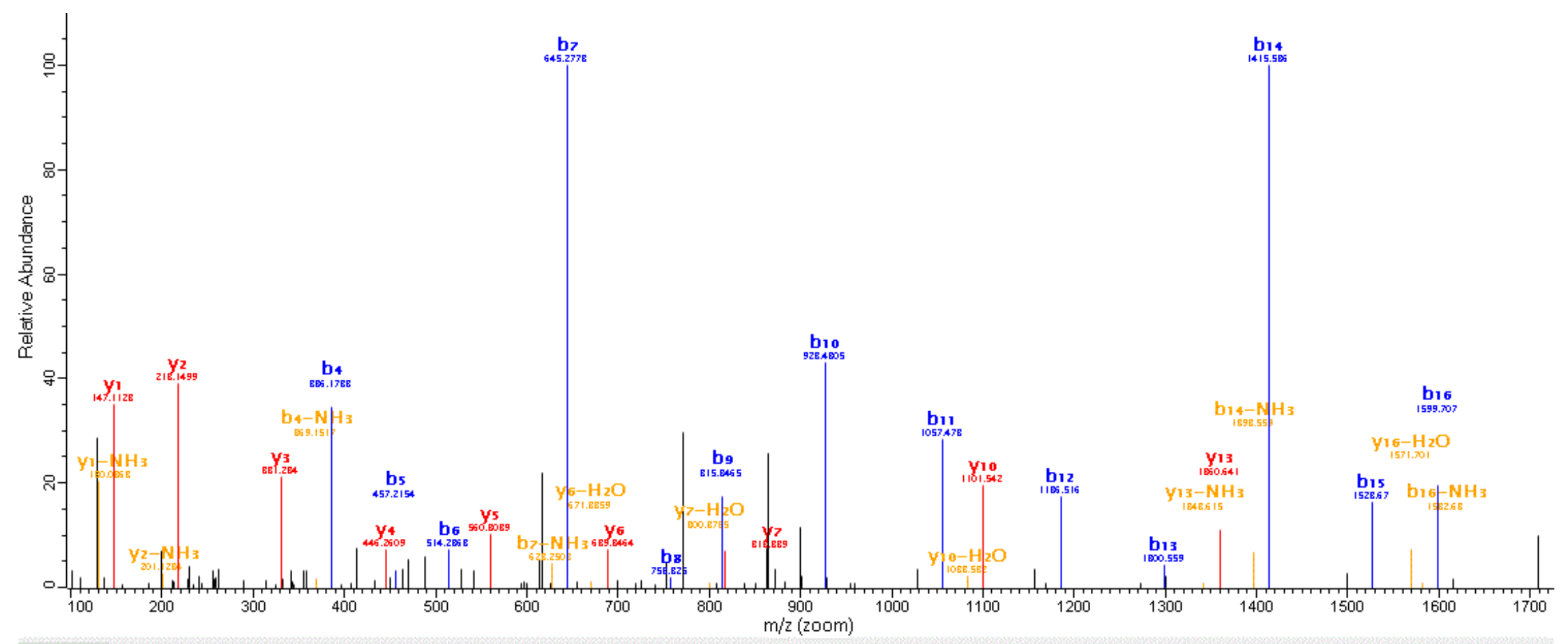

Sequence PSMs Fragments Properties

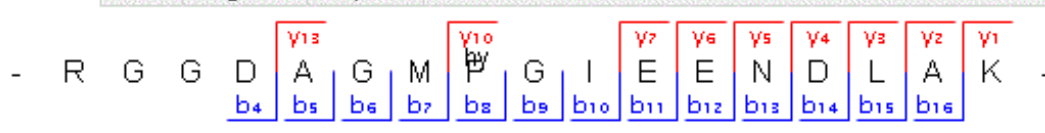

Figure S7. RGGDAGMP[Hydroxylation on P]GIEENDLAK 


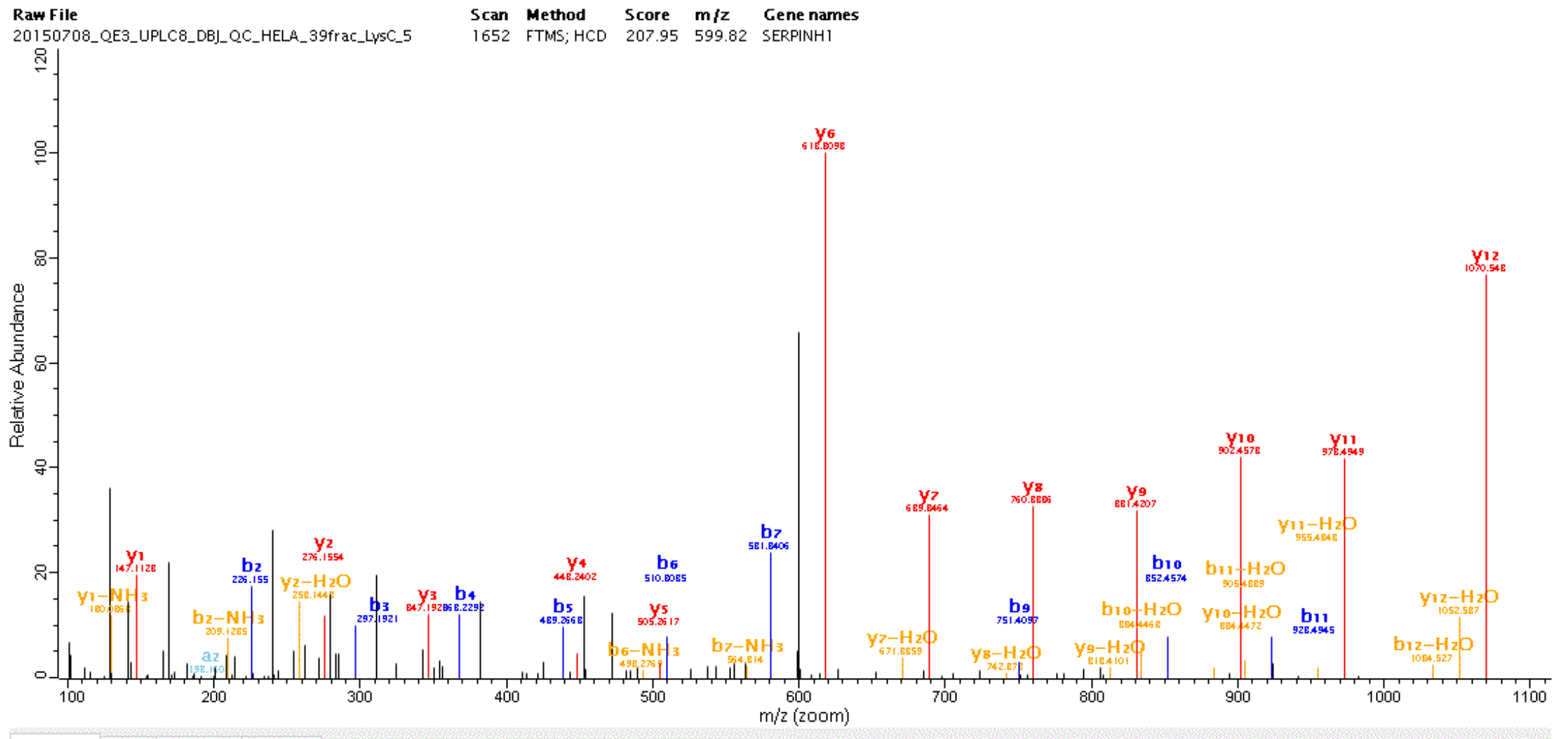

Sequence PSMs Fragments Properties

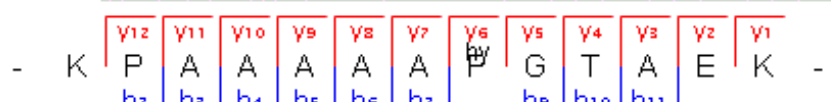

$\underline{b_{2}} b_{3} b_{4} b_{5} b_{6} b_{7} \quad b_{9} b_{10} b_{11}$

Figure S8. KPAAAAAP[Hydroxylation on P]GTAEK.

Page S-9 


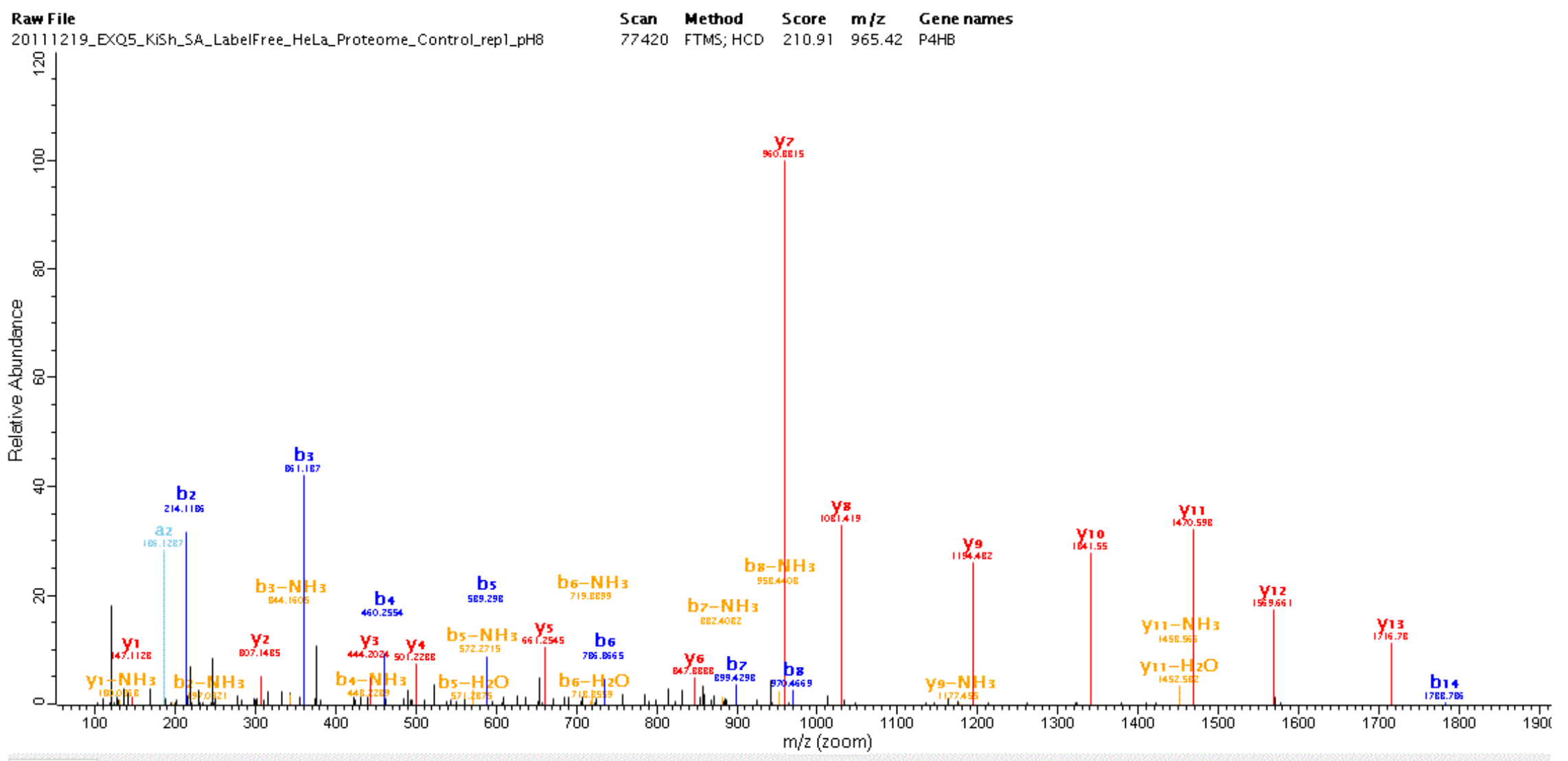

Sequence PSMs Fragments Properties

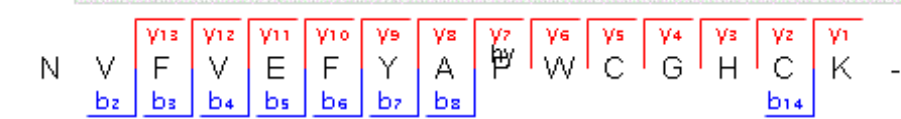

Figure S10. NVFVEFYAP[Hydroxylation on P]WCGHCK. 
Raw File

20111219 _EXOS_KiSh_SA_LabelFree_HeLa_Proteome_Control_rep1_pH5

$$
\approx
$$

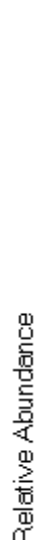

(1)

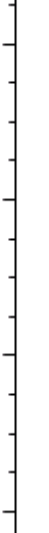

.
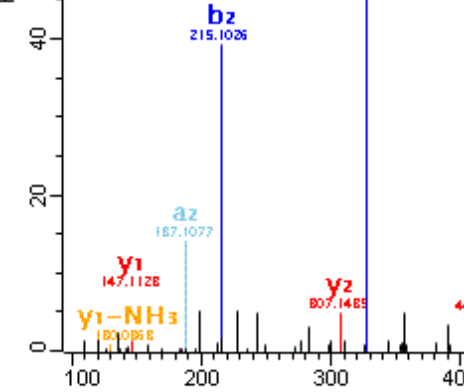

400

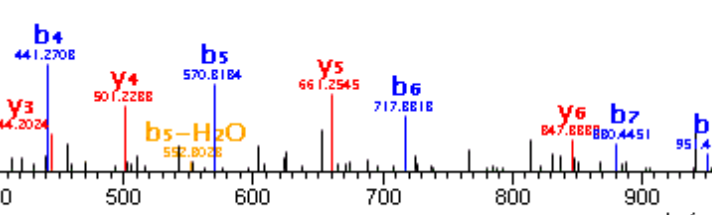

Scan Method Score $\mathrm{m} / \mathrm{z}$ Gene names

82959 FTMS; HCD $168.3 \quad 955.93 \quad$ PDIA4;PDIA3

Sequence PSMs Fragments Properties

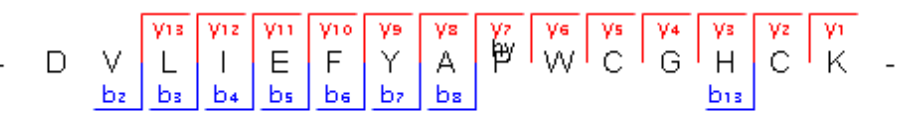

Figure S11. DVLIEFYAP[Hydroxylation on P]WCGHCK.

Page S-11 


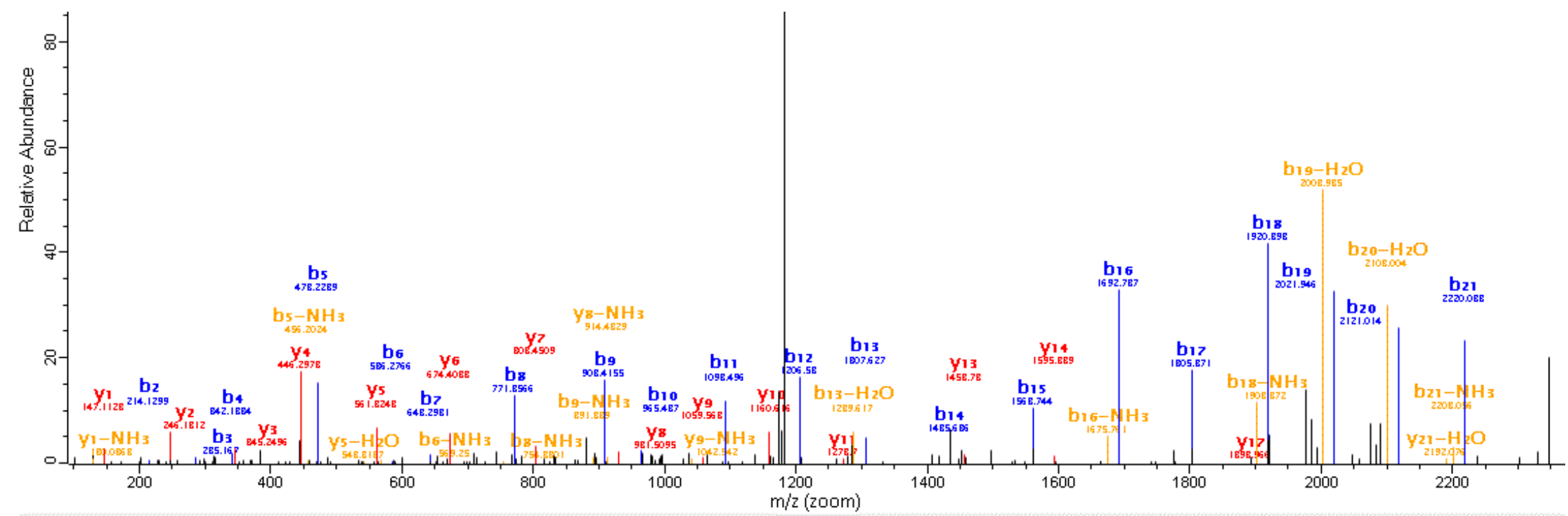

Sequence PSMs Fragments Properties

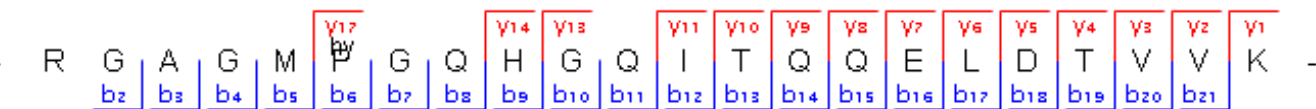

Figure S12. RGAGMP[Hydroxylation on P]GQHGQITQQELDTVVK. 


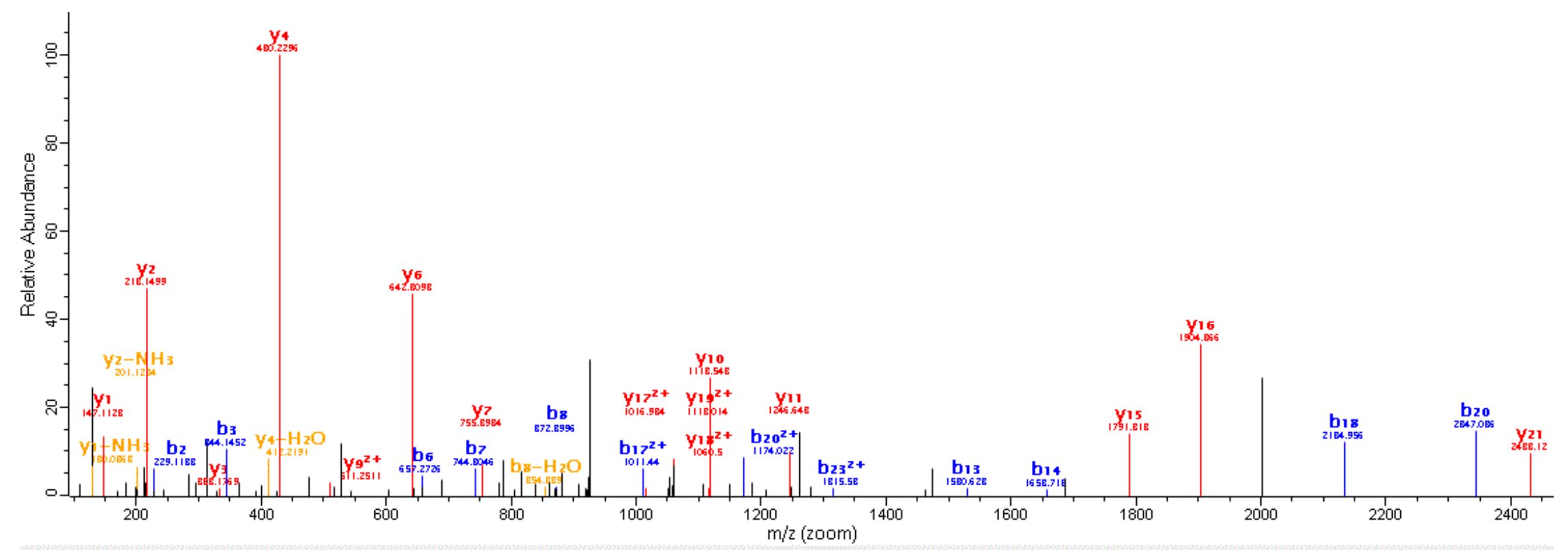

Sequence PSMs Fragments Properties

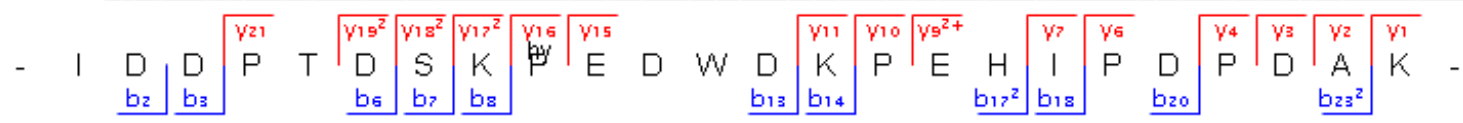

Figure S13. IDDPTDSKP[Hydroxylation on P]EDWDKPEHIPDPDAK. 


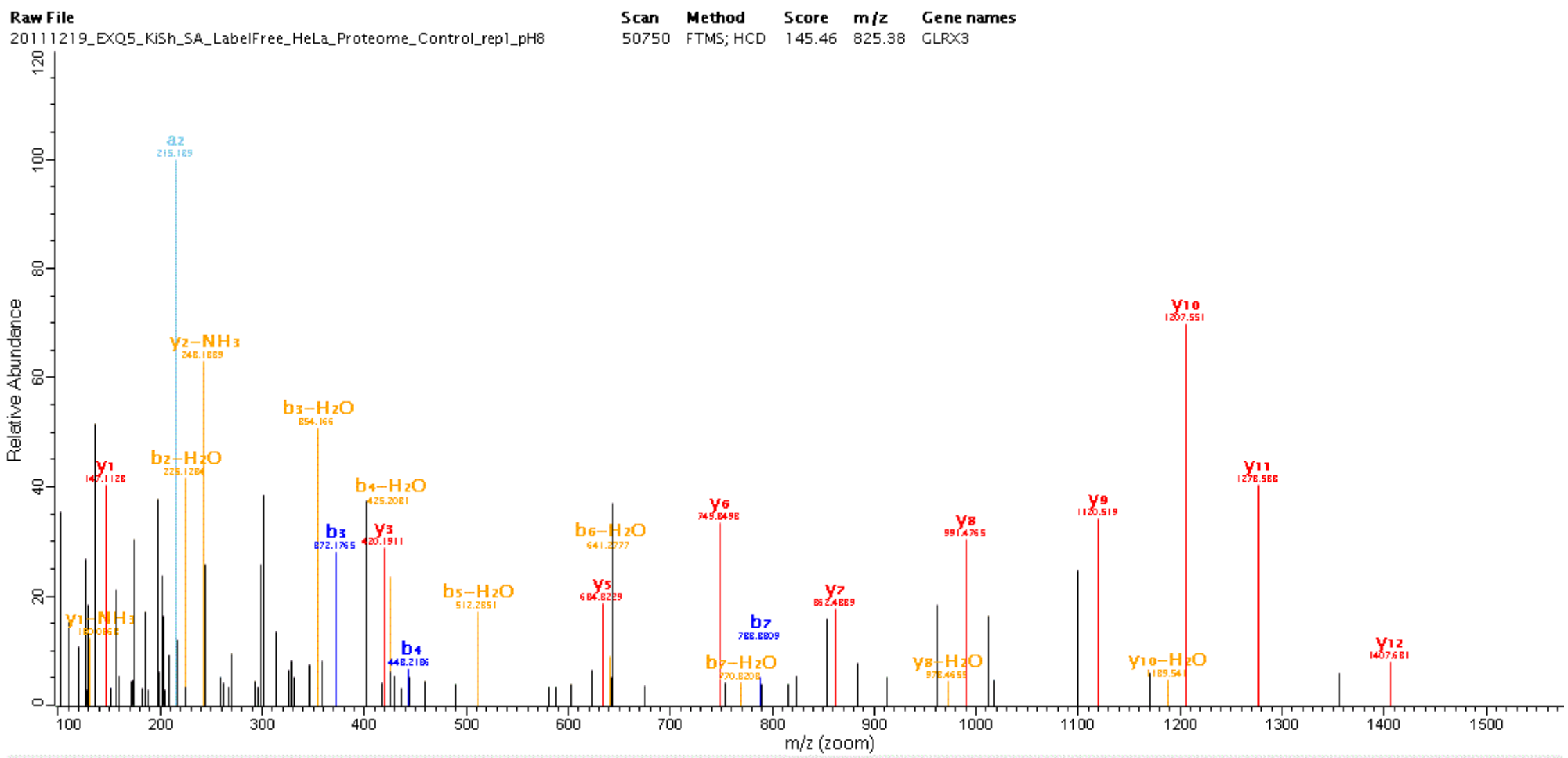

Sequence PSMs Fragments Properties

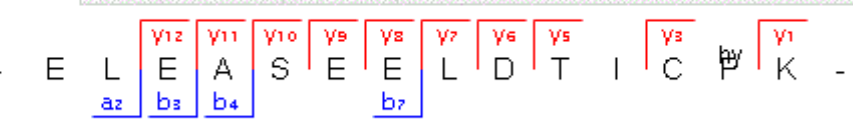

Figure S14. ELEASEELDTICP[Hydroxylation on P]K. 


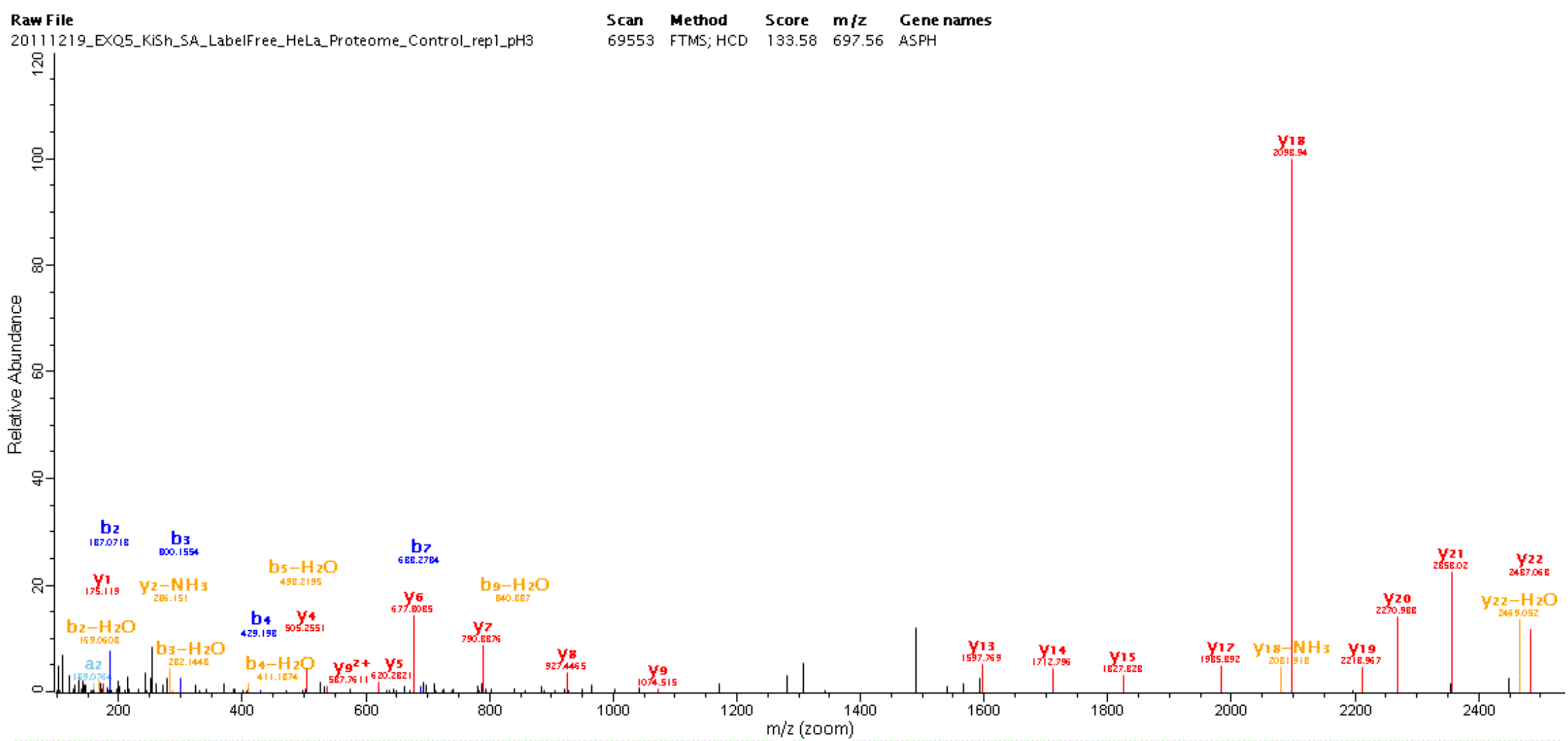

Sequence PSMs Fragments Properties

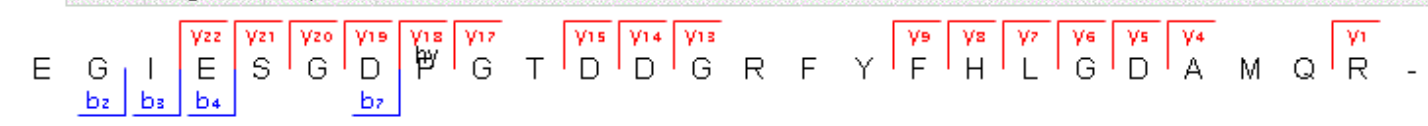

Figure S15. EGIESGDP[Hydroxylation on P]GTDDGRFYFHLGDAMQR. 


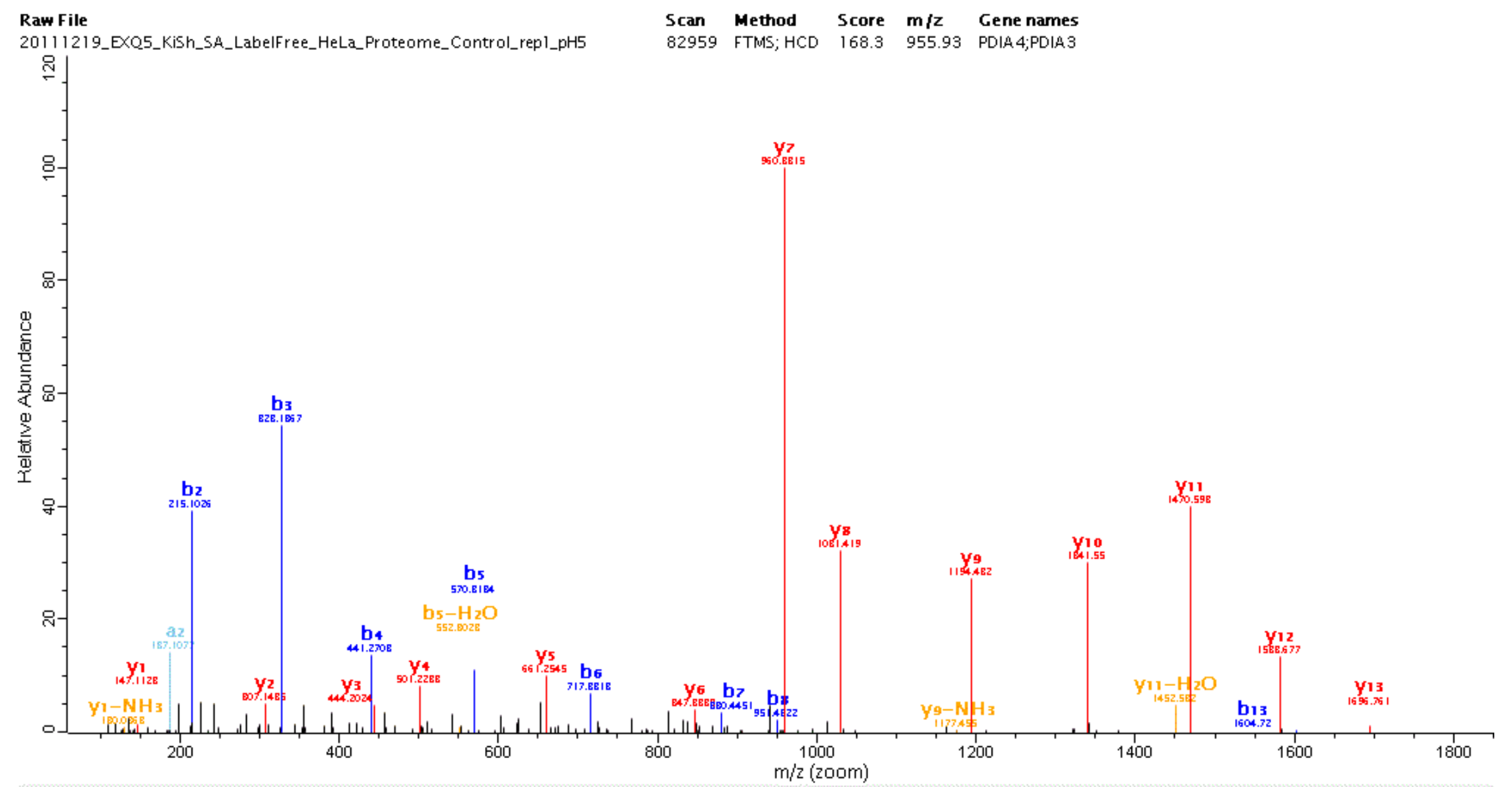

Sequence PSMs Fragments Properties

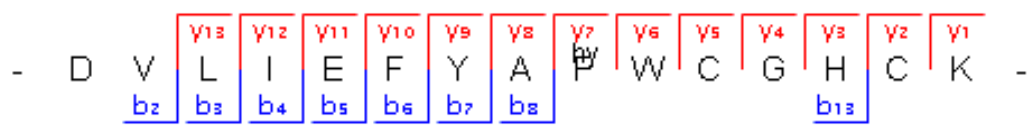

Figure S16. DVLIEFYAP[Hydroxylation on P]WCGHCCK.

Page S-16 


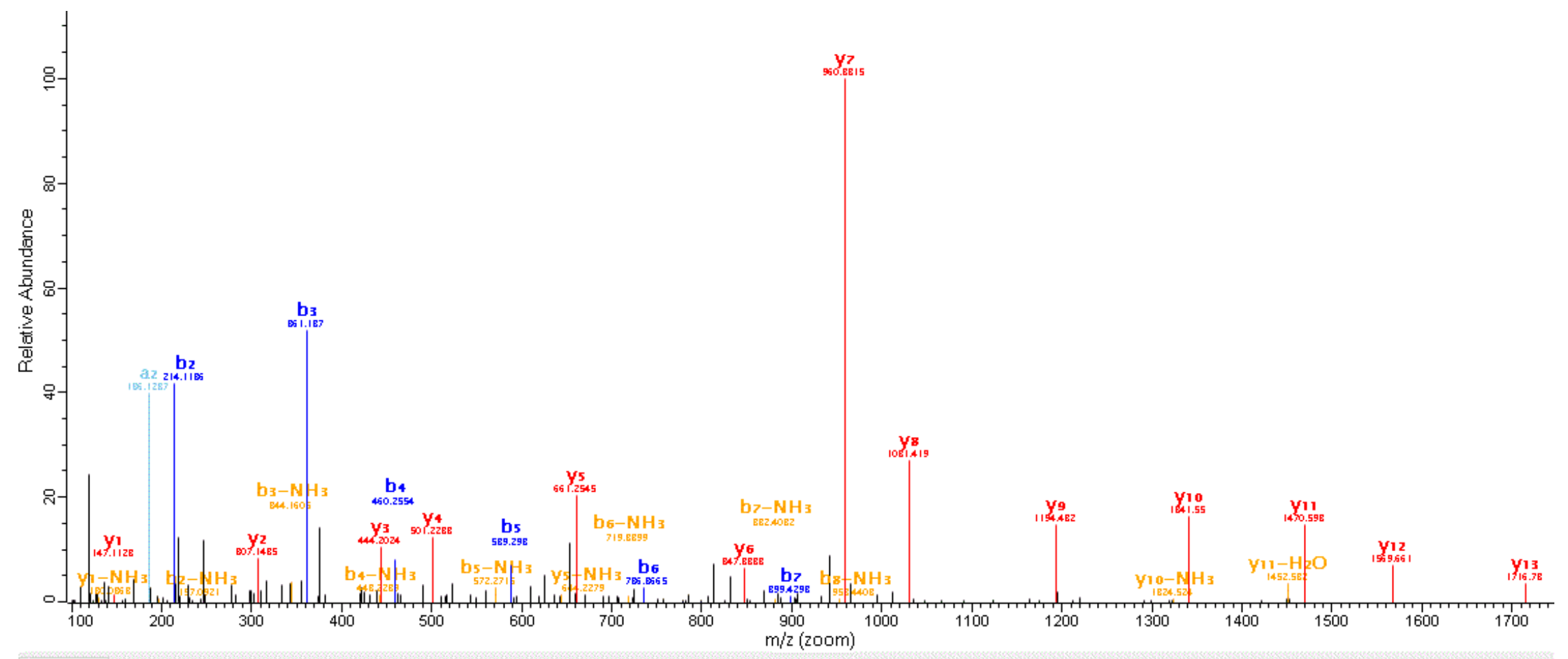

Sequence PSMs Fragments Properties

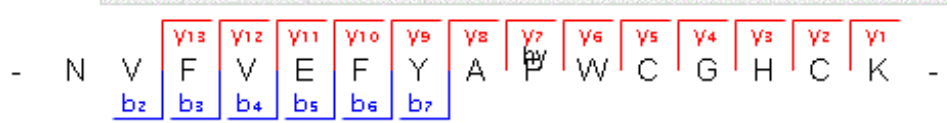

Figure S17. NVFVEFYAP[Hydroxylation on P]WCGHCK. 


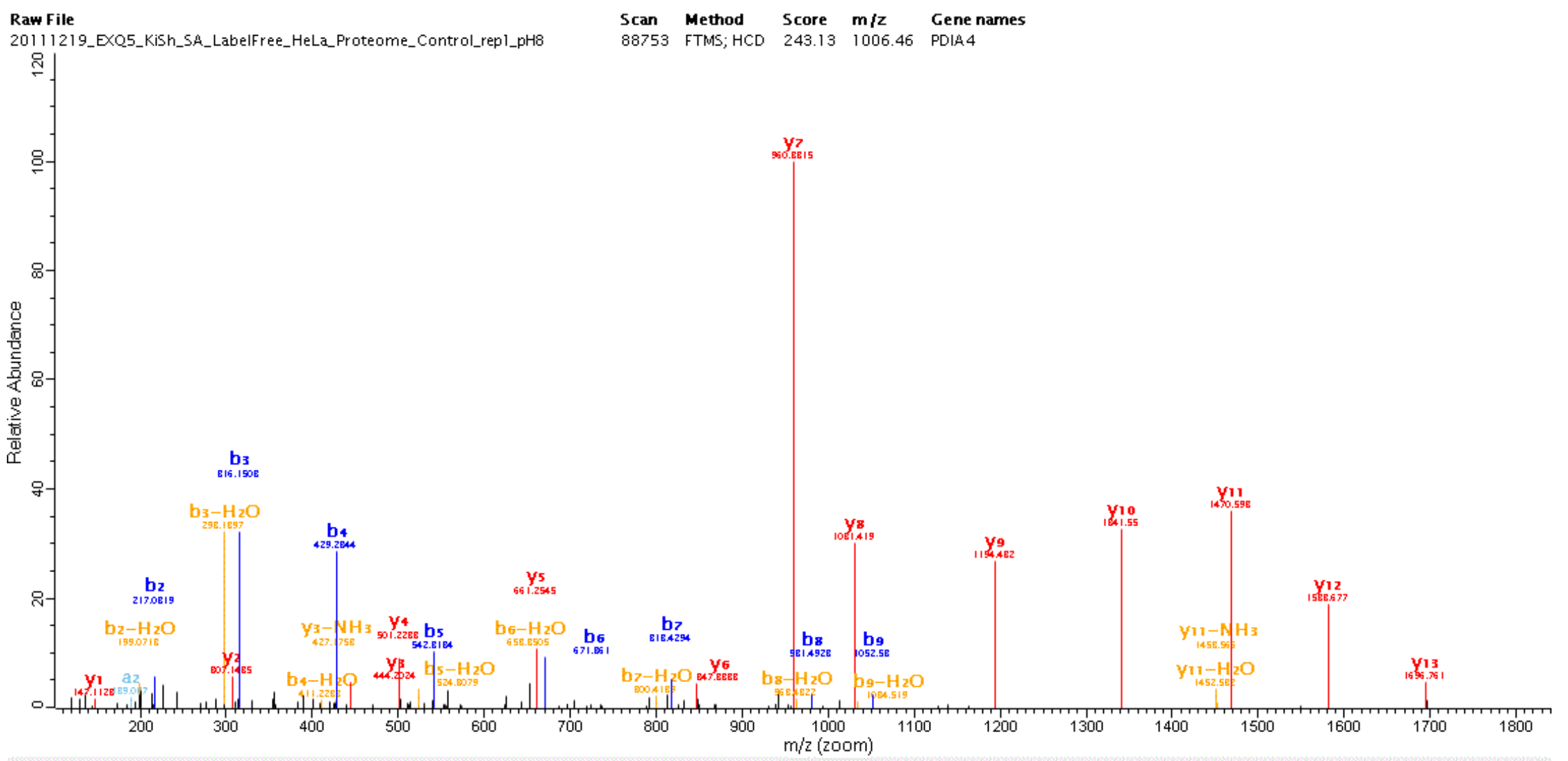

Sequence PSMs Fragments Properties

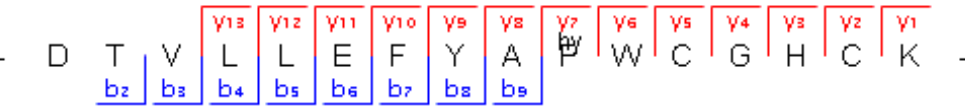

Figure S18. DTVLLEFYAP[Hydroxylation on P]WCGHCK.

Page S-18 


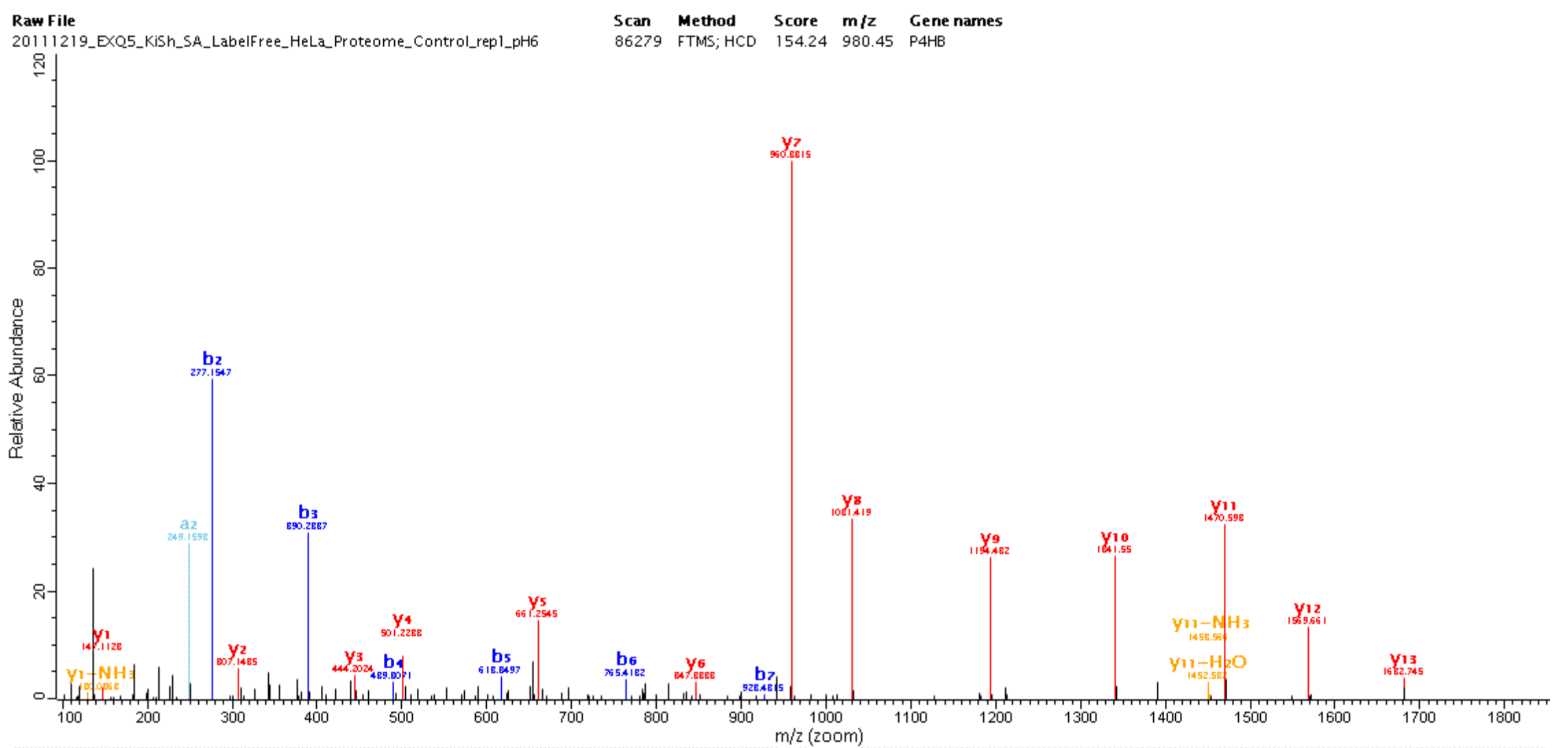

Sequence PSMs Fragments Properties

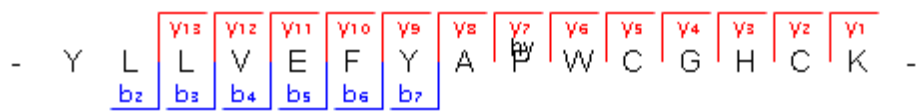

Figure S19. YLLVEFYAP[Hydroxylation on P]WCGHCK.

Page S-19 


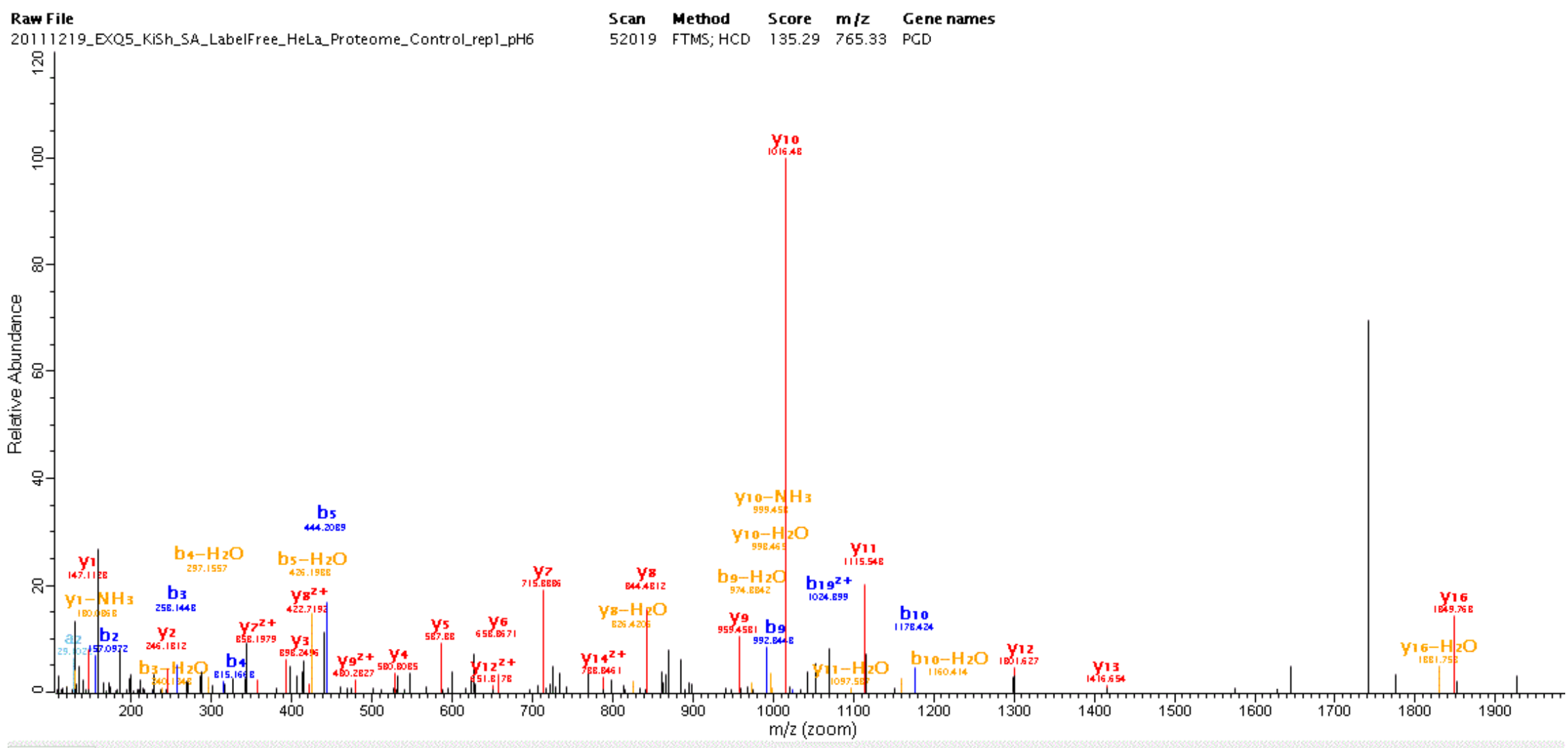

Sequence PSMs Fragments Properties

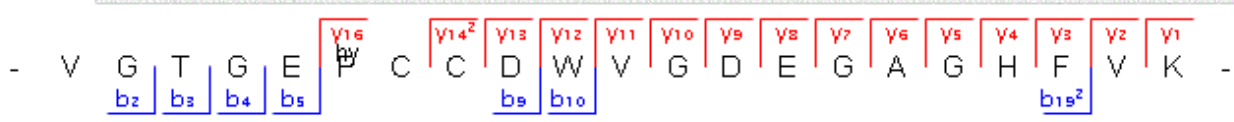

Figure S20. VGTGEP[Hydroxylation on P]CCDWVGDEGAGHFVK.

Page S-20 
Raw File

2011121 _EXO5_KiSh_SA_LabelFree_HeLa_Proteome_Control_repl_pH3

$\stackrel{2}{2}$

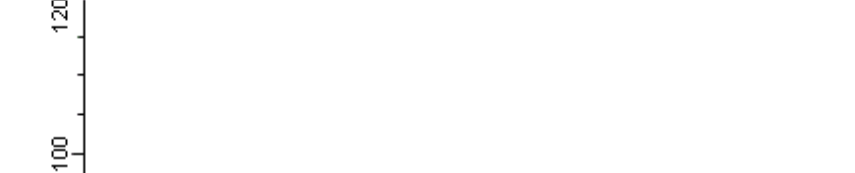

Scan Method Score $\mathrm{m} / \mathrm{z}$ Gene names

39545 FTMS; HCD $168.56 \quad 695.07 \quad$ CALR

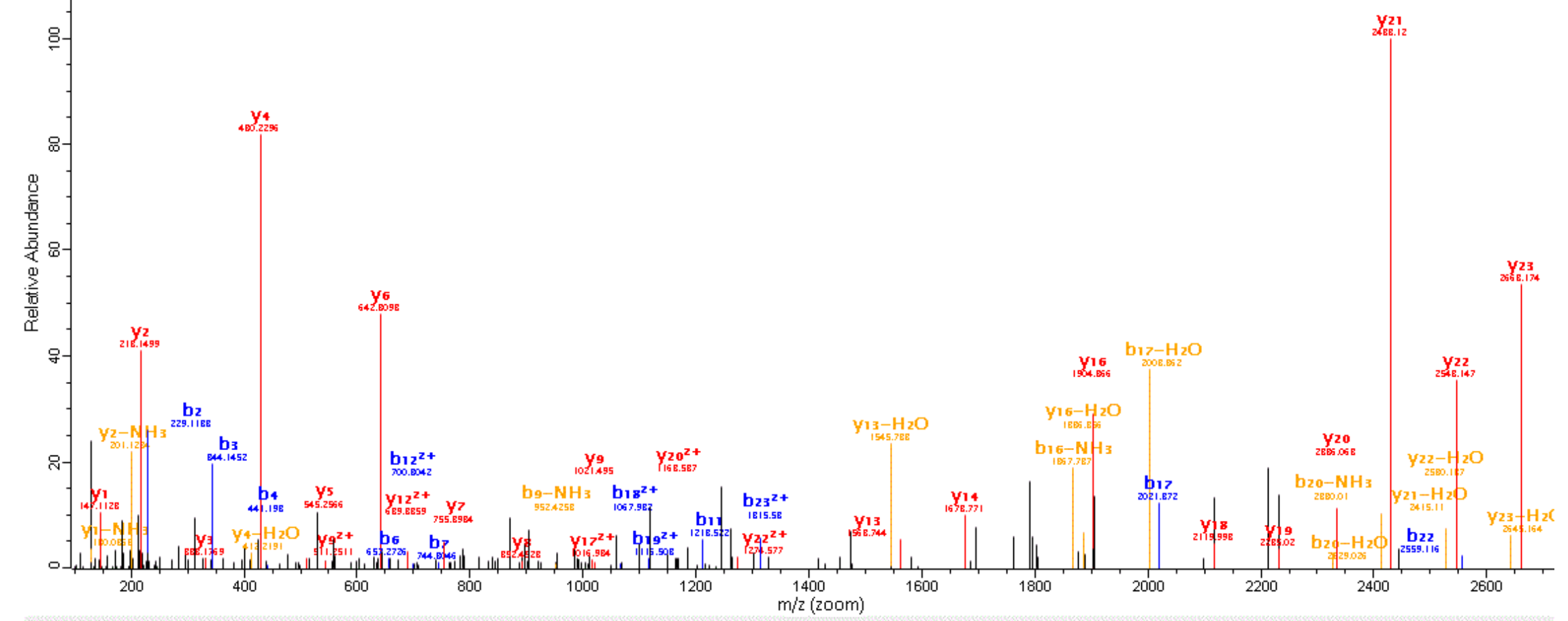

Sequence PSMs Fragments Properties

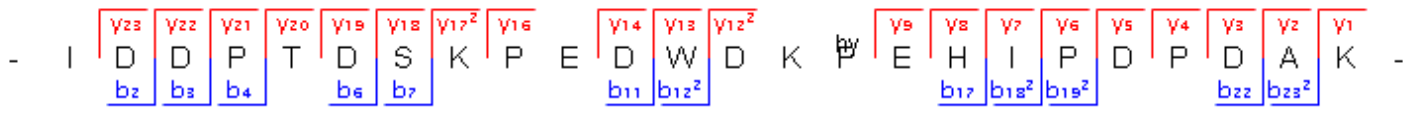

Figure S21. IDDPTDSKP[Hydroxylation on P]EDWDKPEHIPDPDAK.

Page S-21 


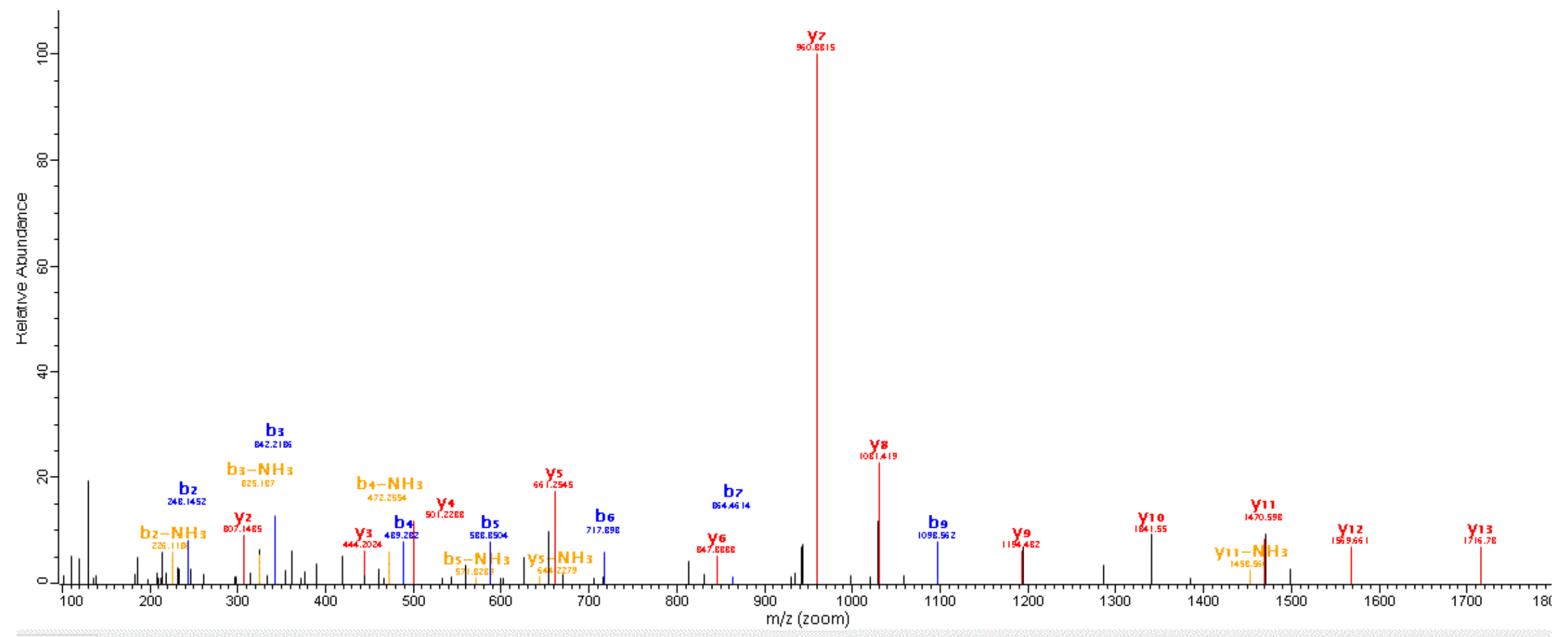

Sequence PSMs Fragments Properties

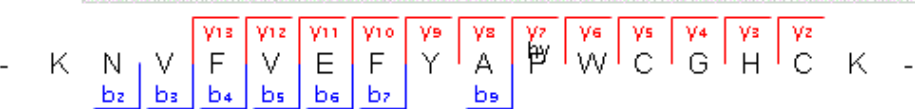

Figure S22. KNVFVEFYAP[Hydroxylation on P]WCGHCK. 


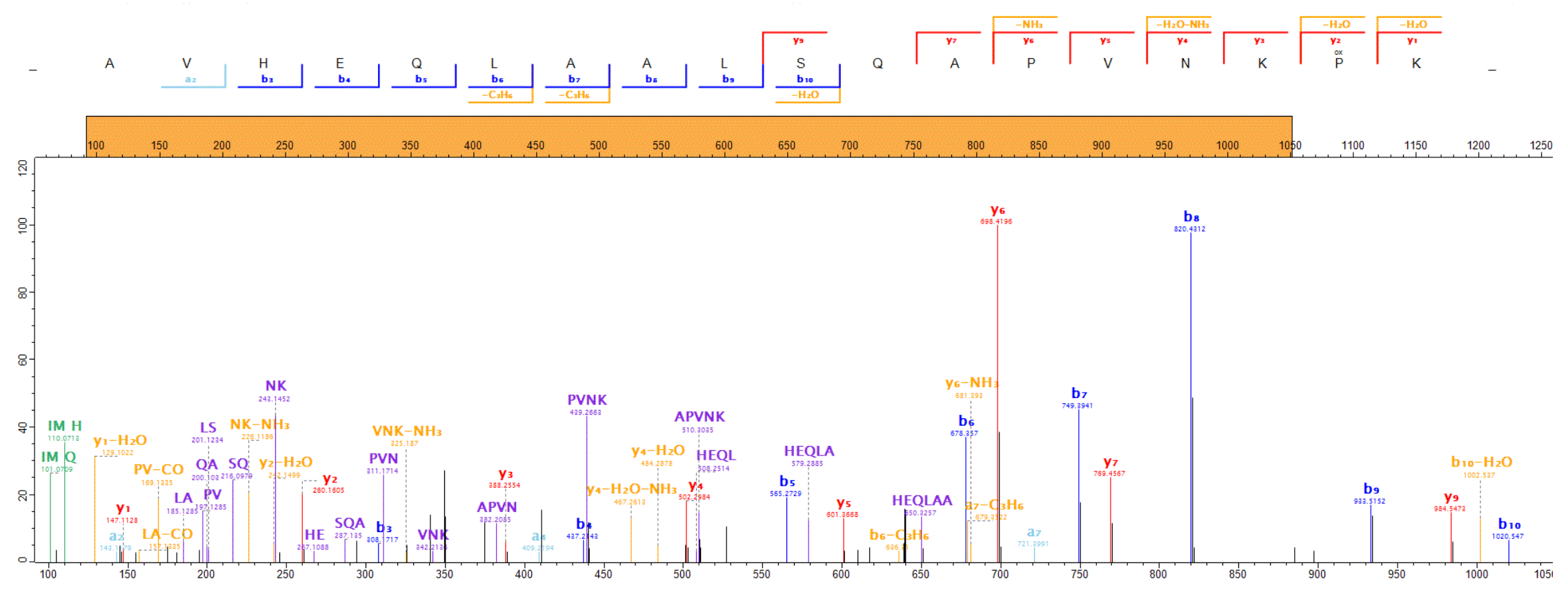

Figure S23. AVHEQLAALSQAPVNNKP[Hydroxylation on P]K. 


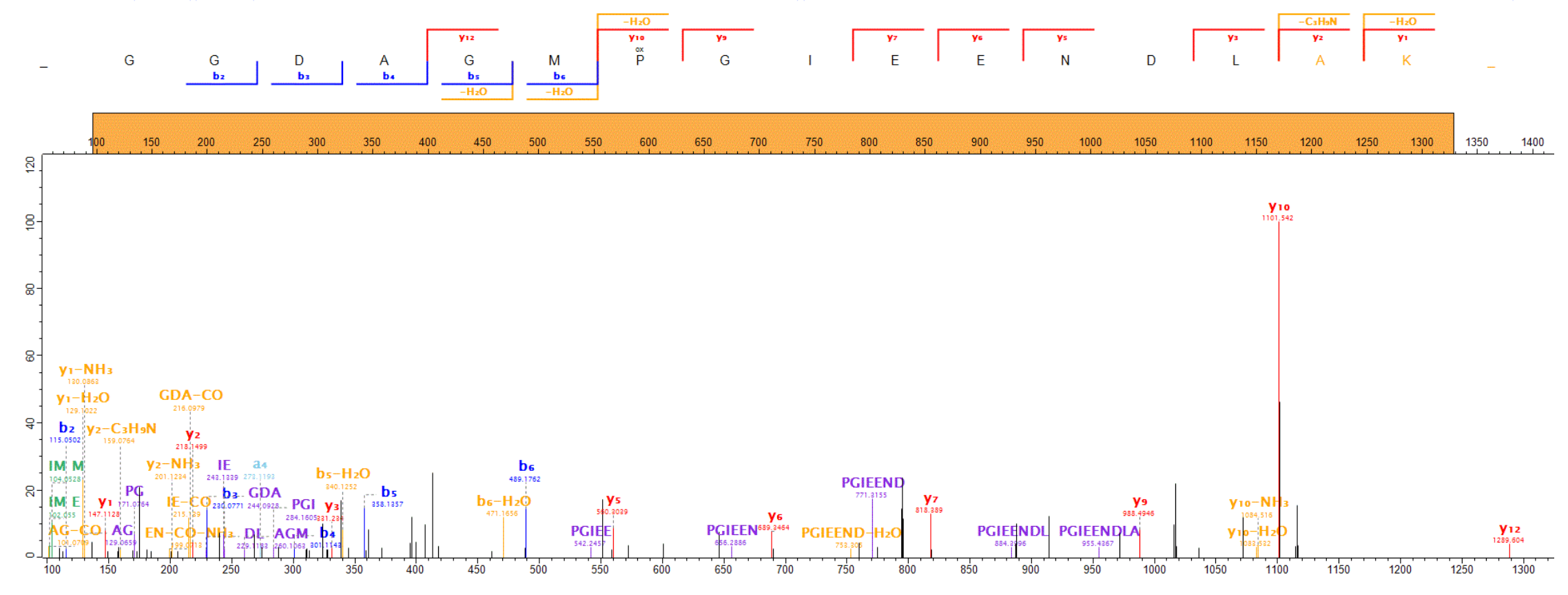

Figure S24. GGDAGMP[Hydroxylation on P]GIEENDLAK. 


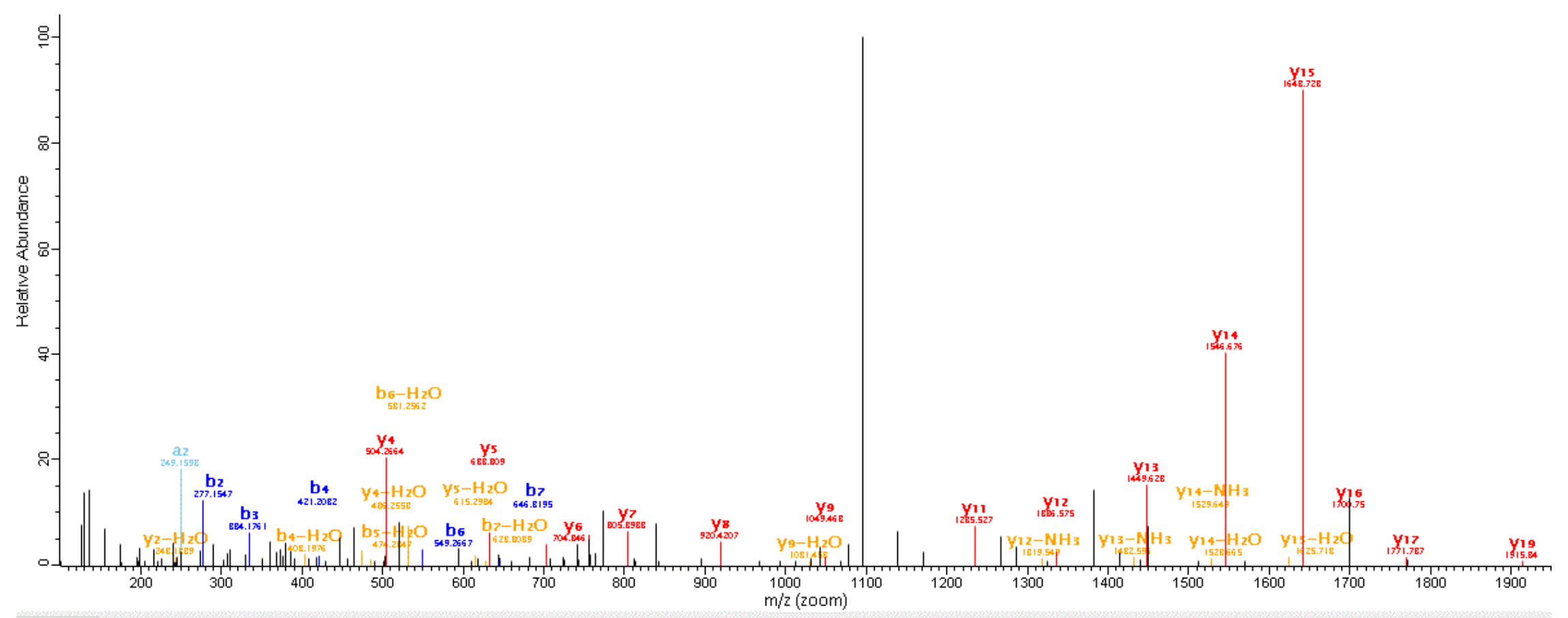

Sequence PSMs Fragments Properties

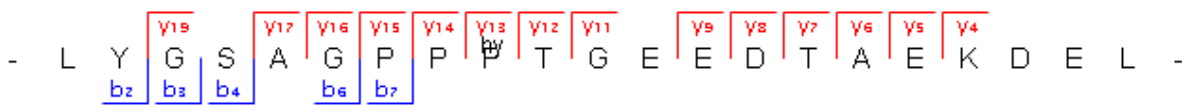

Figure S25. LYGSAGPPP[Hydroxylation on P]TGEEDTAEKDEL. 


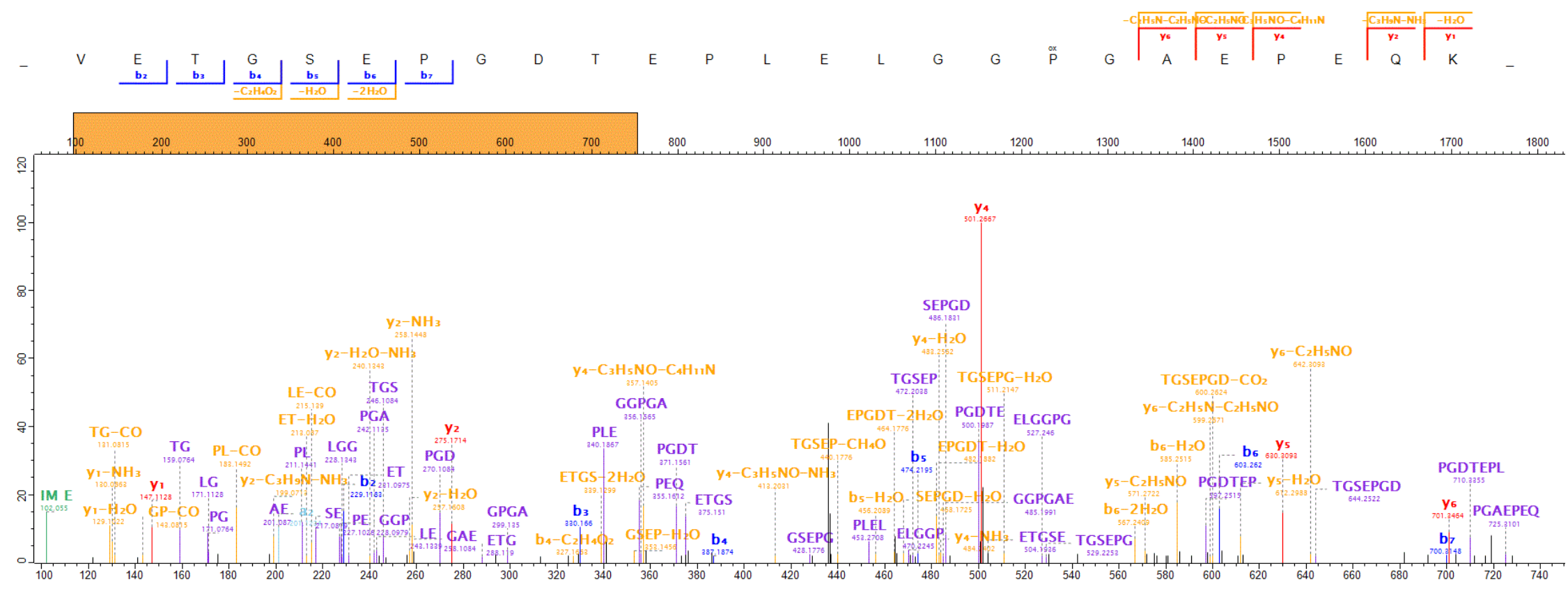

Figure S26a. VETGSEPGDTEPLELGGP[Hydroxylation on P]GAEPEQK_m/z_100-740. 


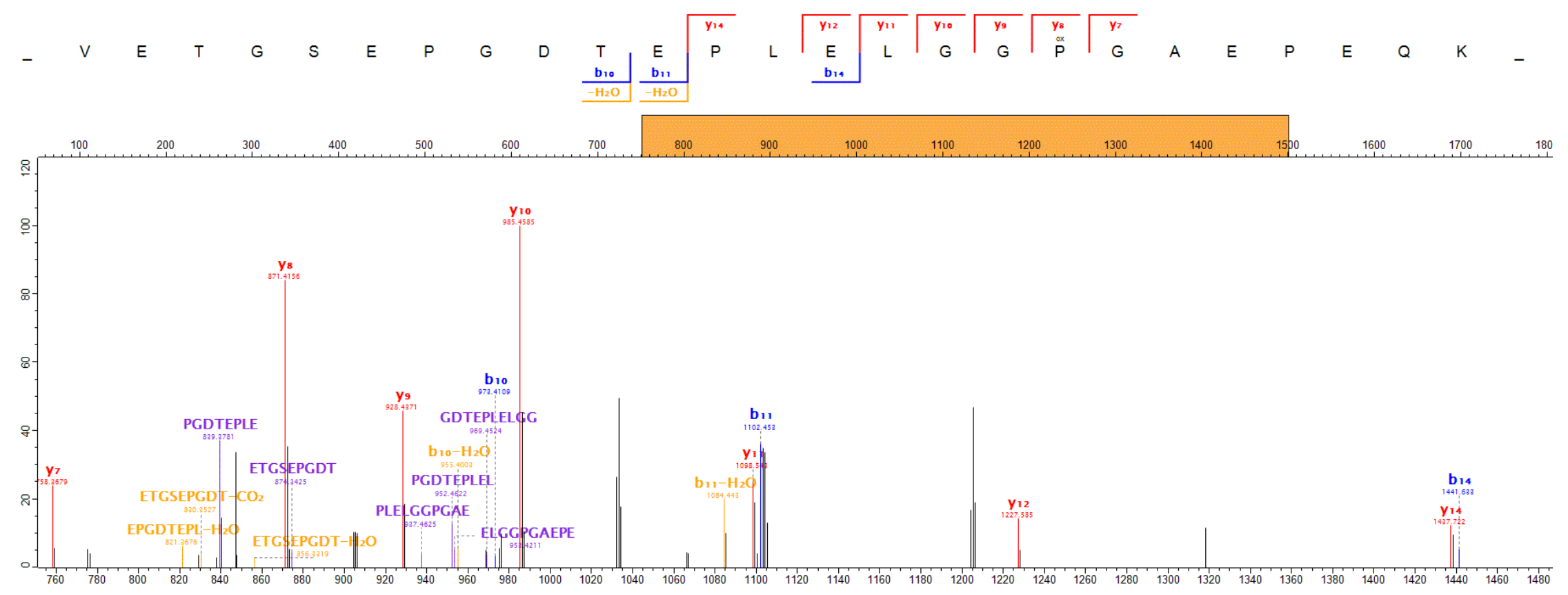

Figure S26b. VETGSEPGDTEPLELGGP[Hydroxylation on P]GAEPEQK_m/z_750-1480. 


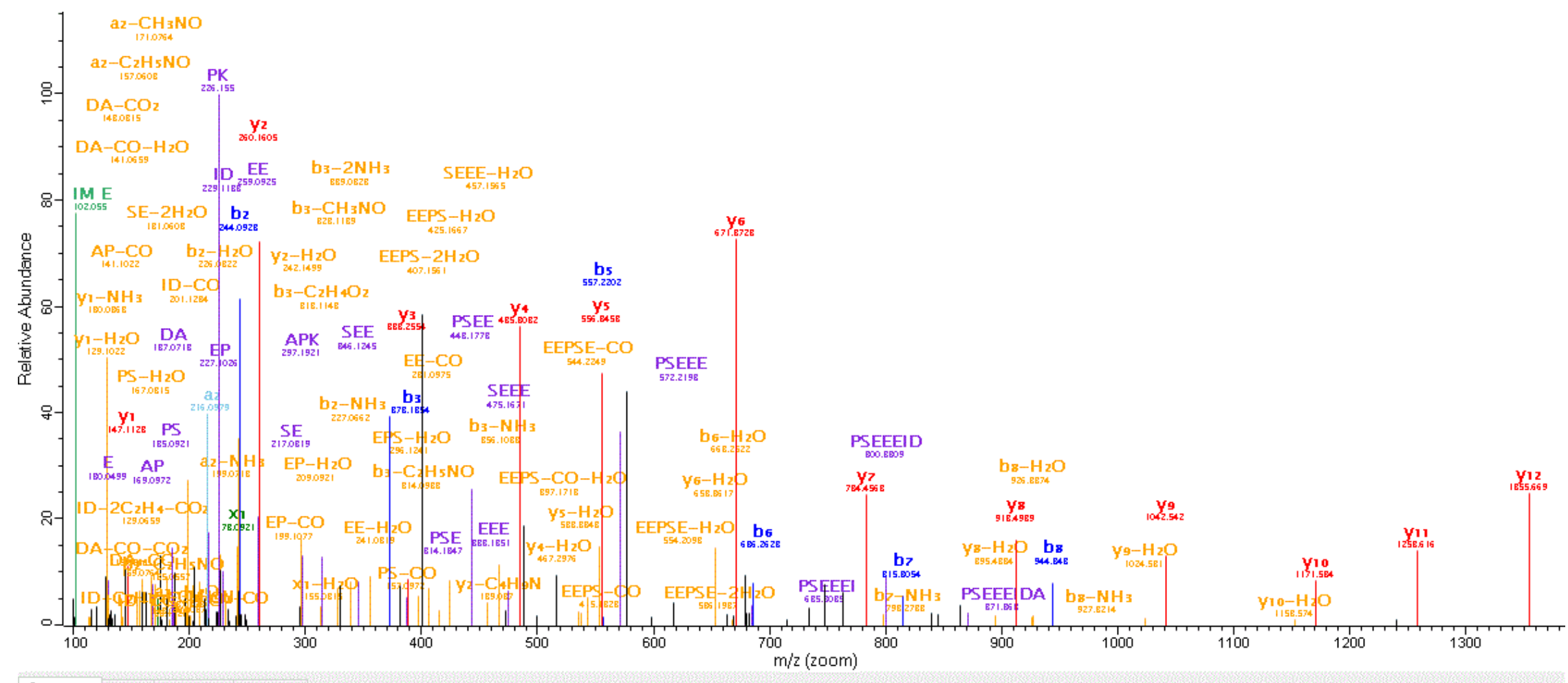

Sequence PSMs Fragments Properties

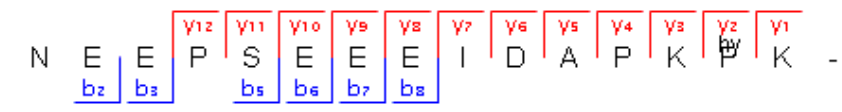

Figure S27. NEEPSEEEIDAPKP[Hydroxylation on P]K. 


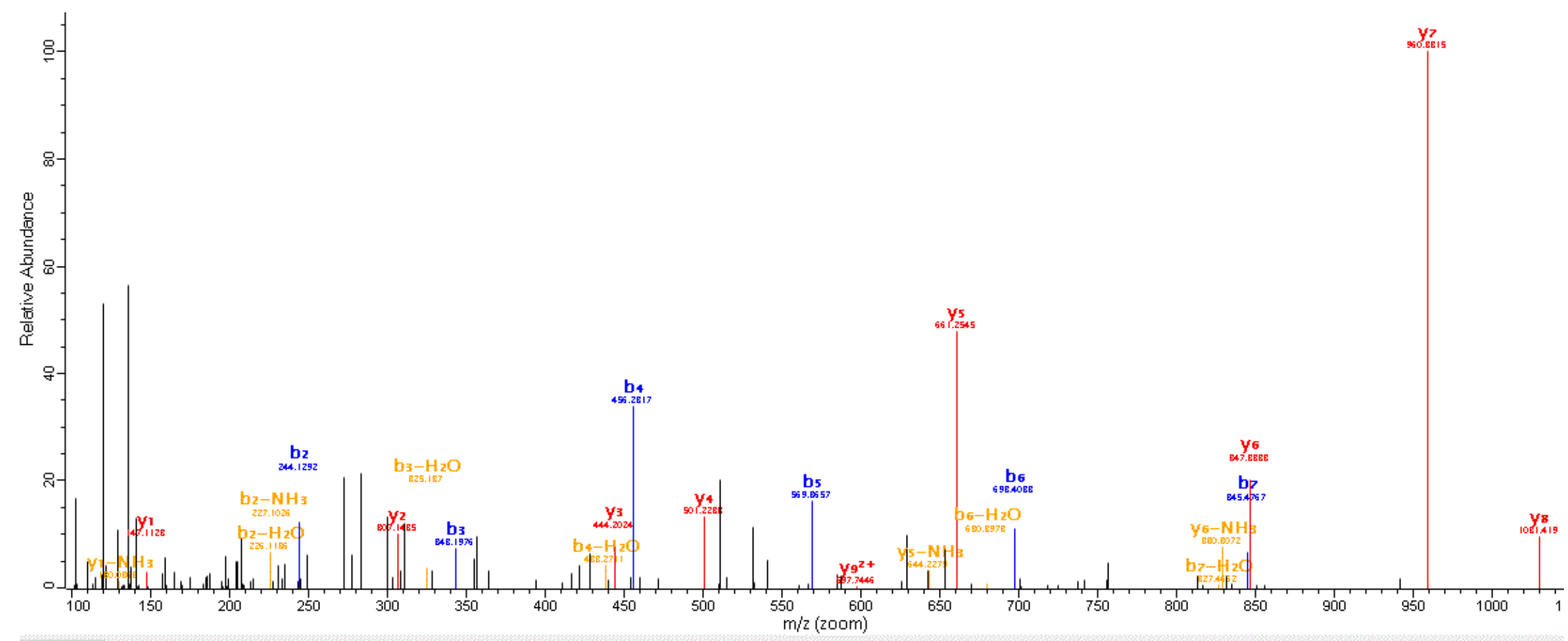

Sequence PSMs Fragments Properties

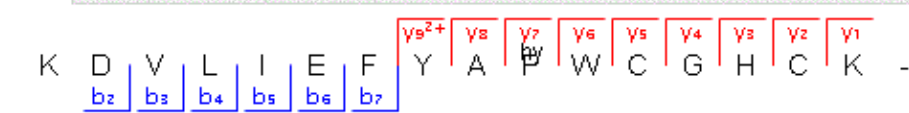

Figure S28. KDVLIEFYAP[Hydroxylation on P]WCGHCK. 


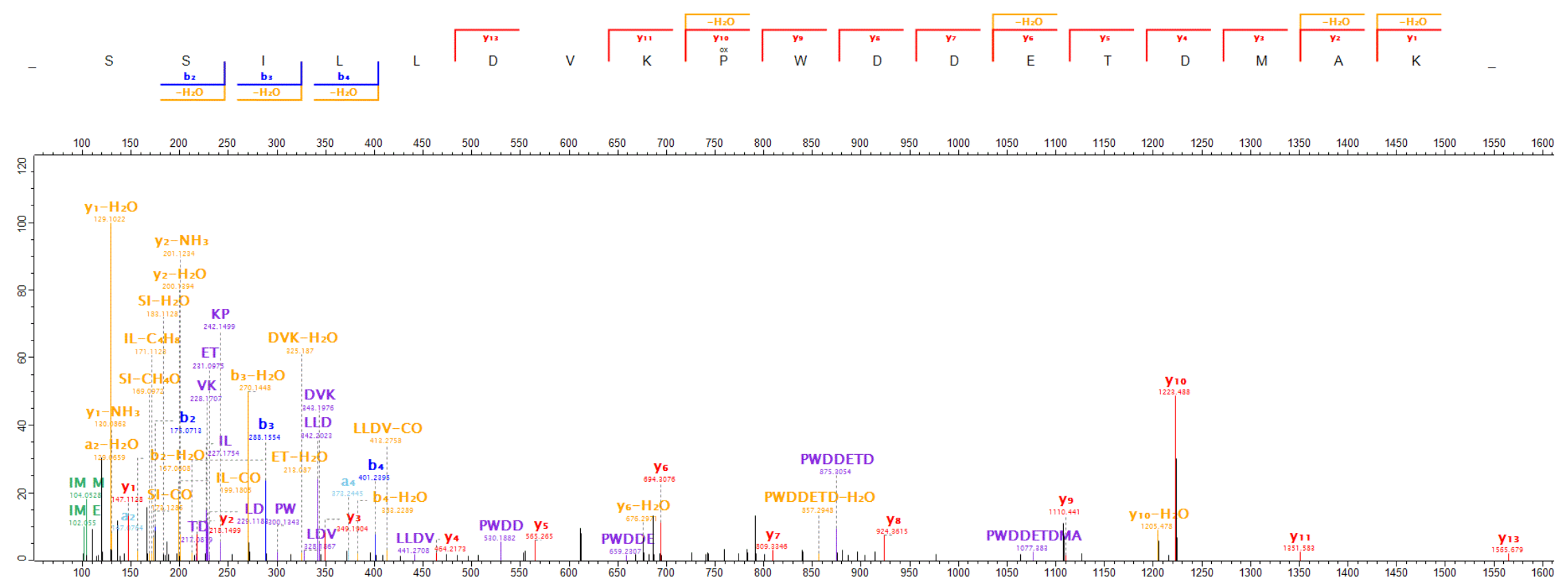

Figure S29. SSILDVKP[Hydroxylation on P]WDDETDMAK. 


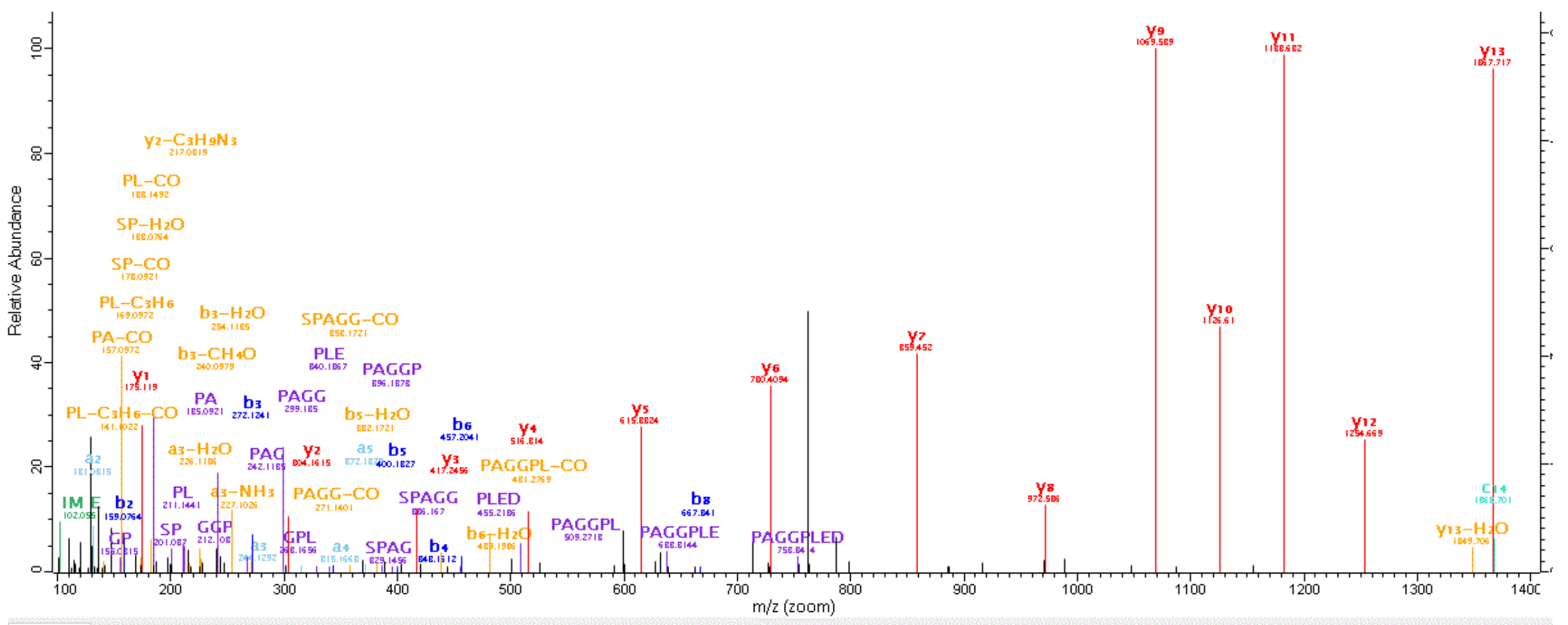

Sequence PSMs Fragments Properties

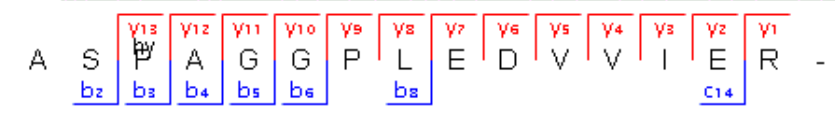

Figure S30. ASP[Hydroxylation on P]AGGPLEDVVIER. 


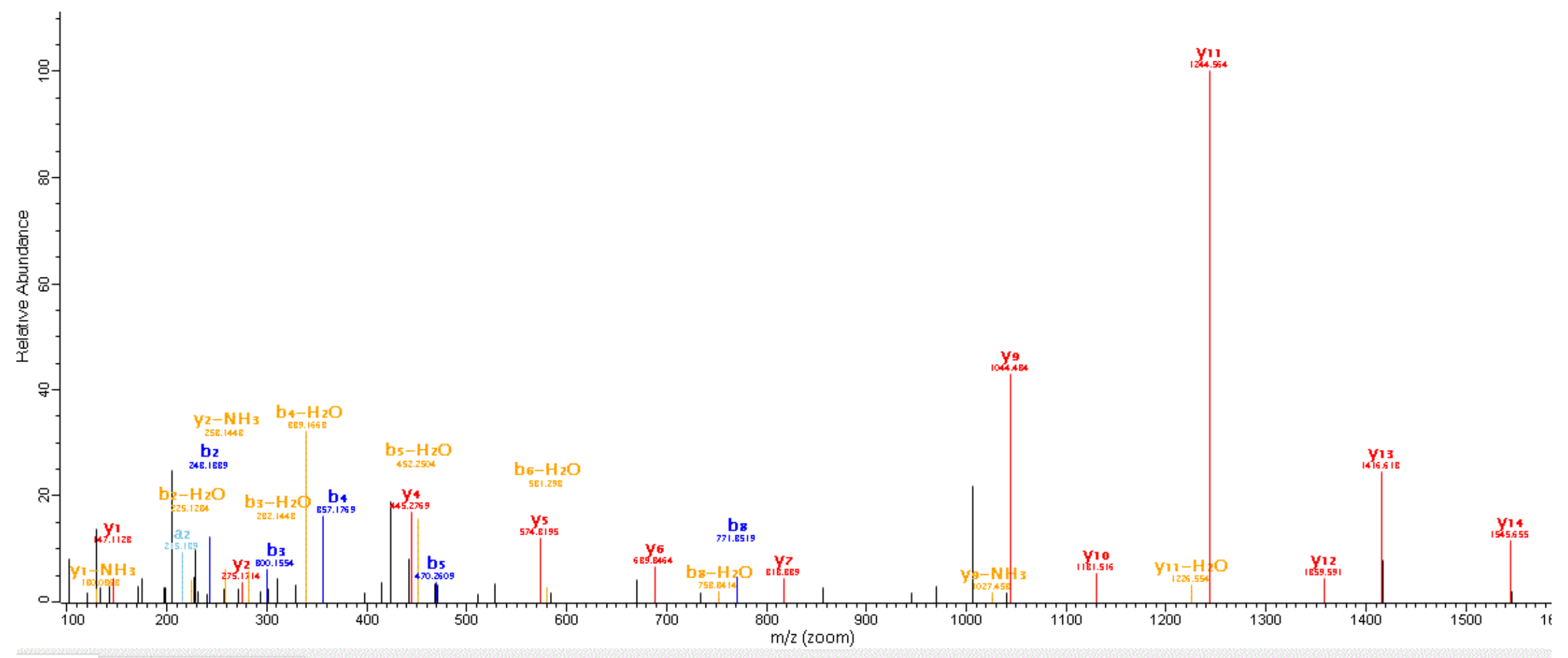

Sequence PSMS Fragments Properties

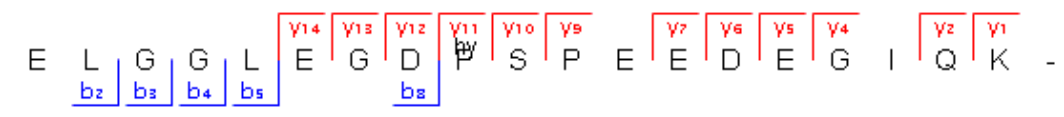

Figure S31. ELGGLEGDP[Hydroxylation on P]SPEEDEGIQK. 


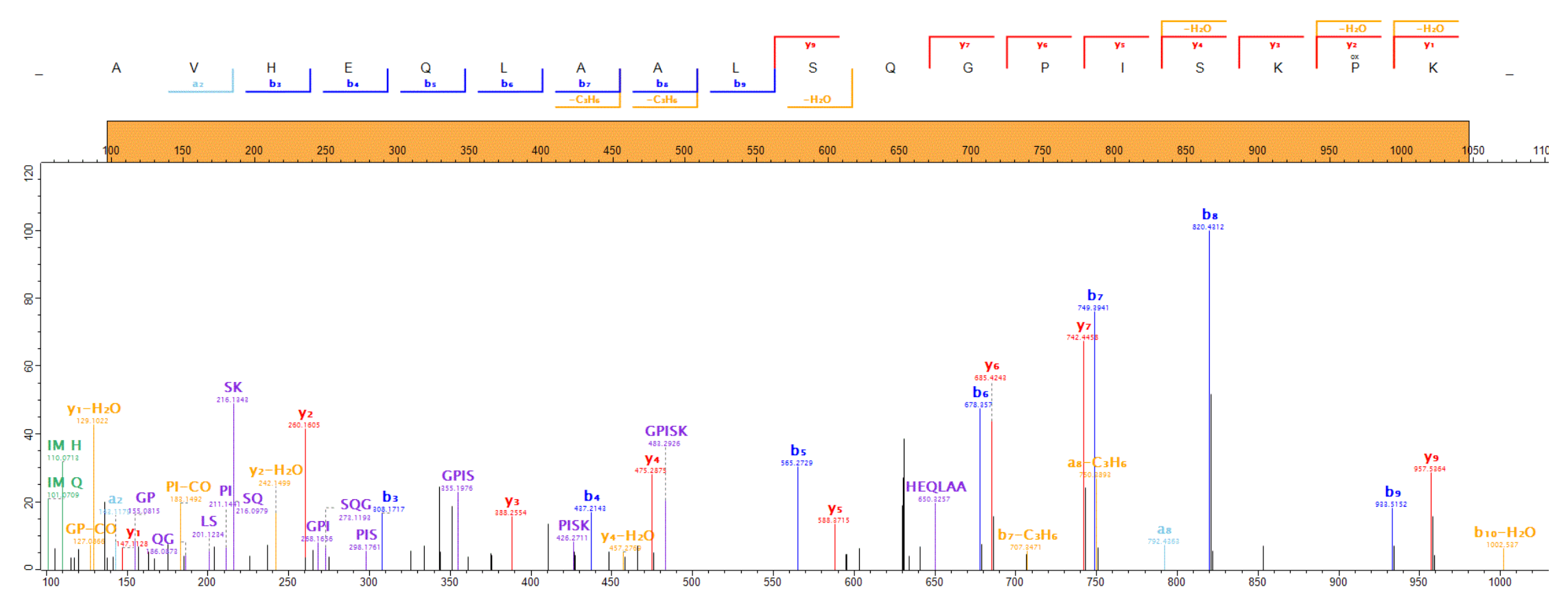

Figure S32. AVHEQLAALSQGPISKP[Hydroxylation on P]K. 


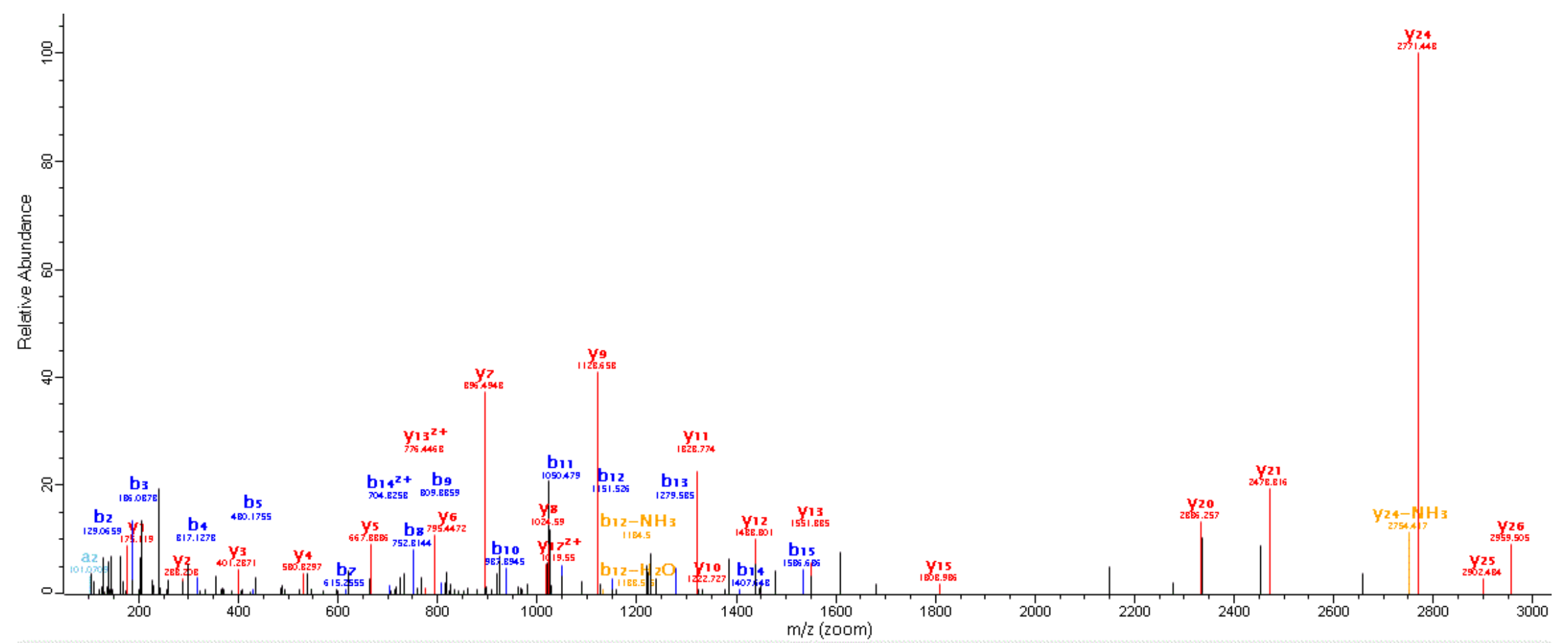

Sequence PSMs Fragments Properties

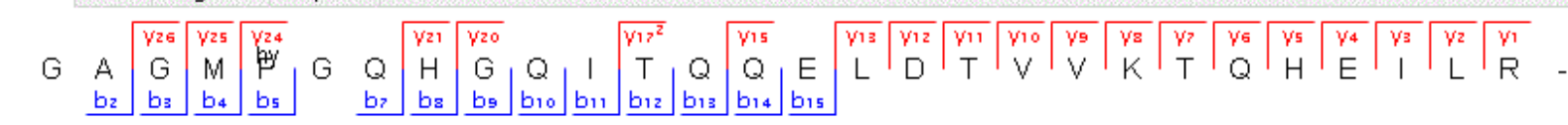

Figure S33. GAGMP[Hydroxylation on P]GQHGQITQQELDTVVKTQHEILR. 


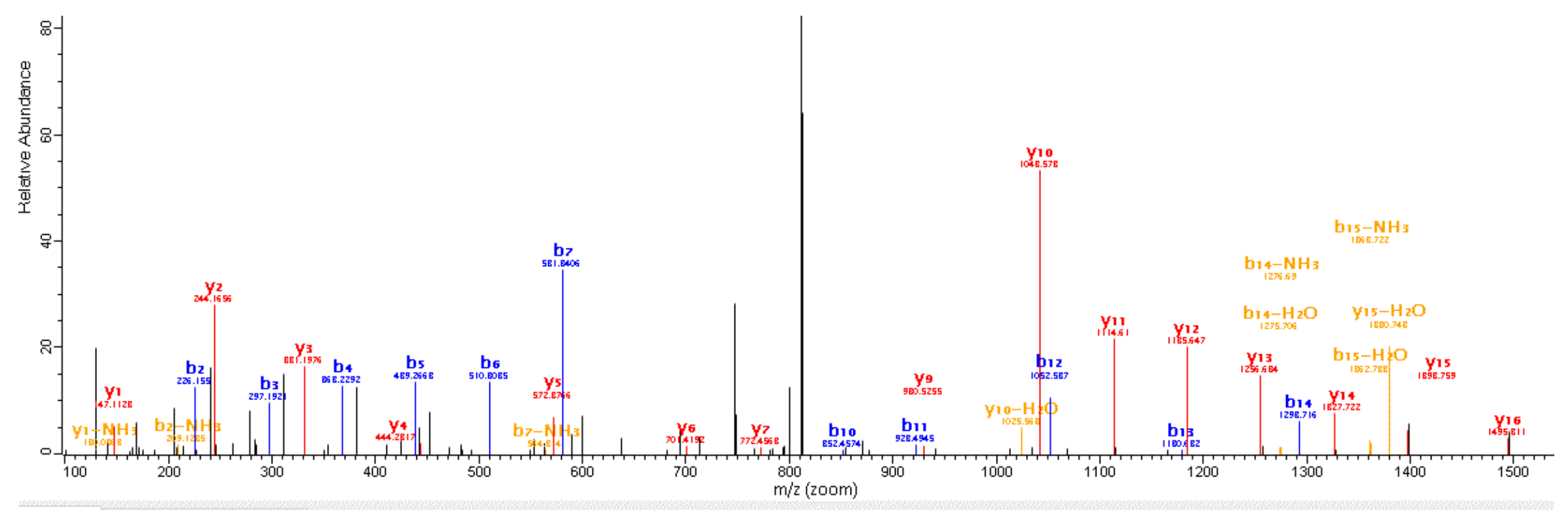

Sequence PSMs Fragments Properties

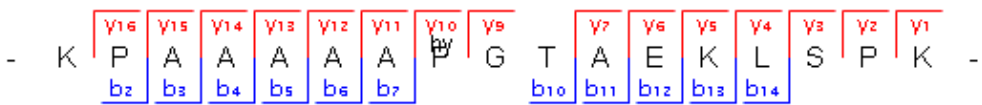

Figure S34. KPAAAAAP[Hydroxylation on P]GTAEKLSPK. 


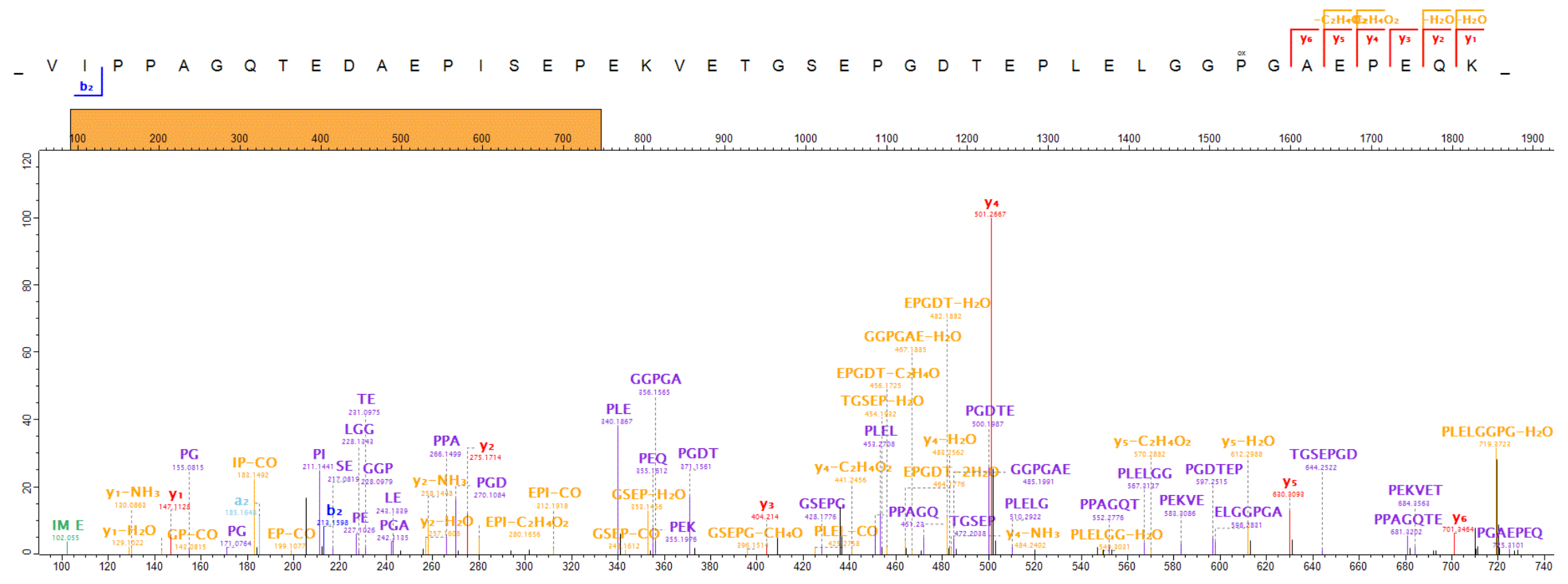

Figure S35a. VIPPAGQTEDAEPISEPEKVETGSEPGDTEPLELGGP[Hydroxylation on P]GAEPEQK_m/z_100-740. 


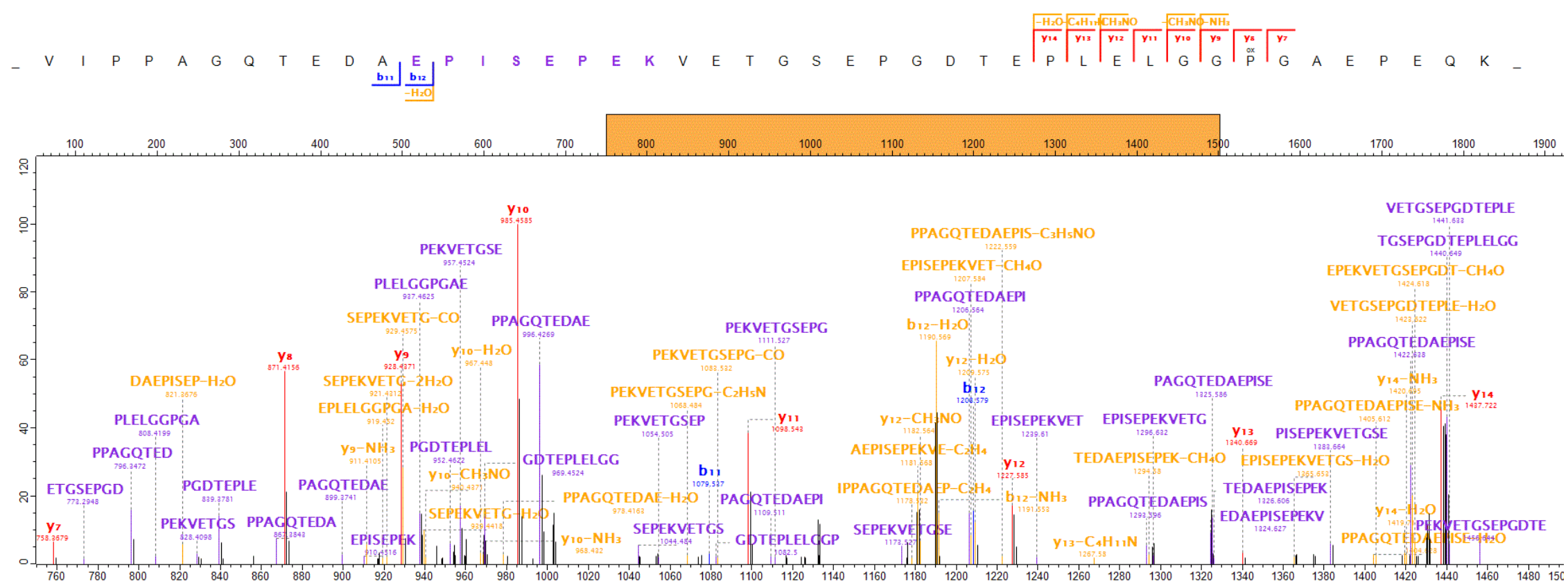

Figure S35b. VIPPAGQTEDAEPISEPEKVETGSEPGDTEPLELGGP[Hydroxylation on P]GAEPEQK_m/z_740-1500. 


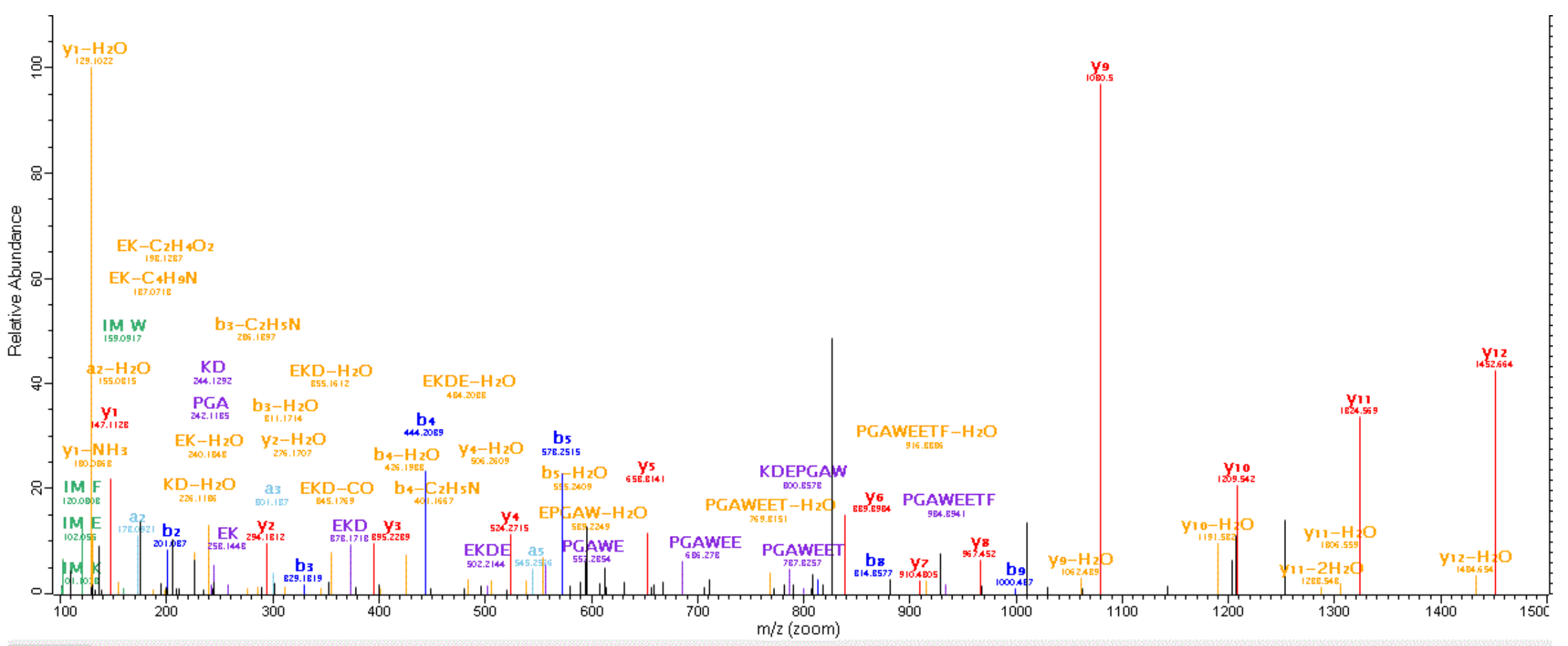

Sequence PSMs Fragments Properties

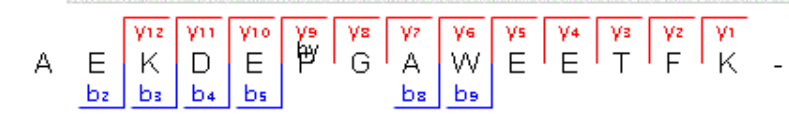

Figure S36. AEKDEP[Hydroxylation on P]GAWEETFK. 


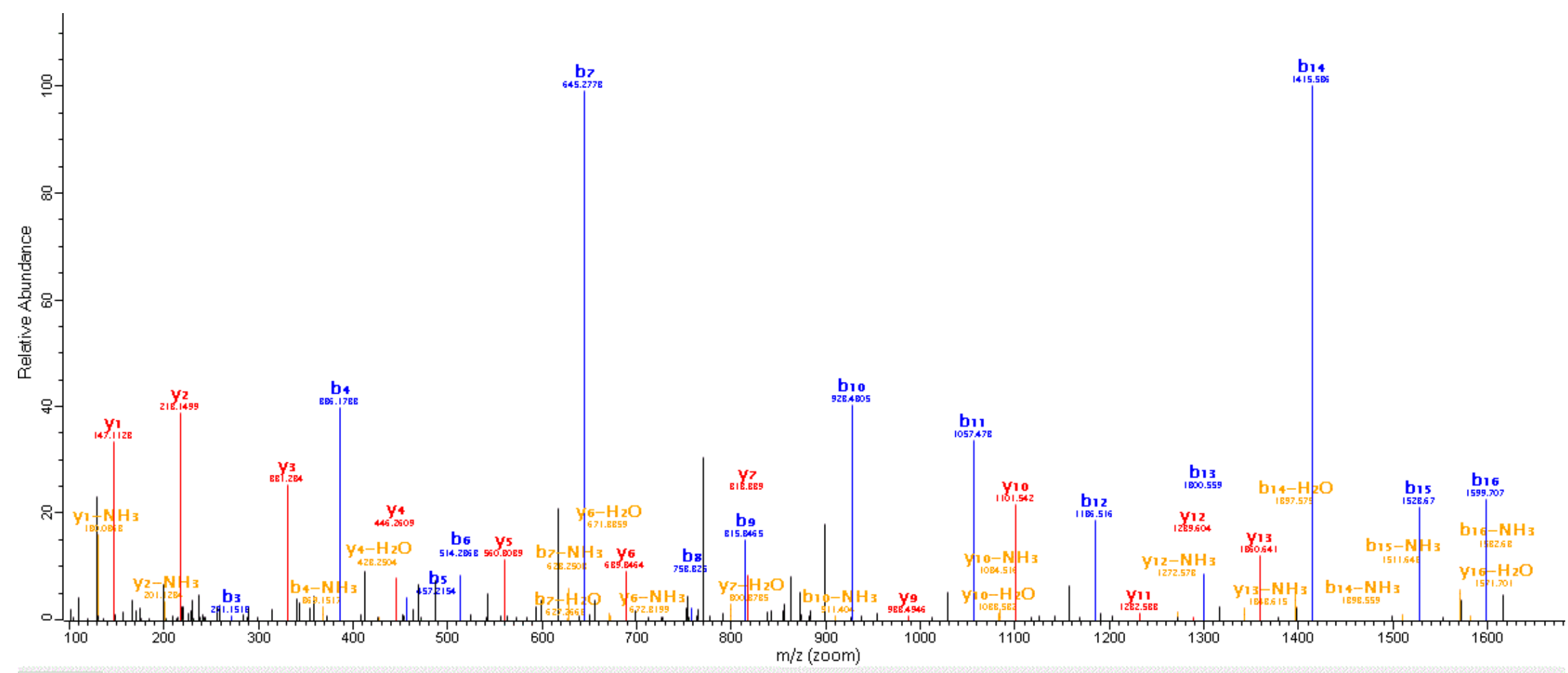

Sequence PSMS Fragments Properties

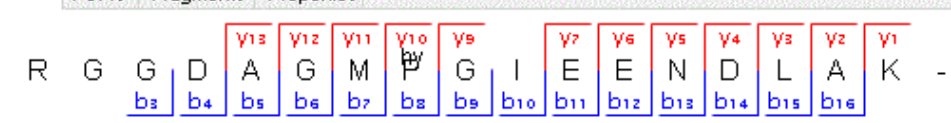

Figure S37. RGGDAGMP[Hydroxylation on P]GIEENDLAK. 


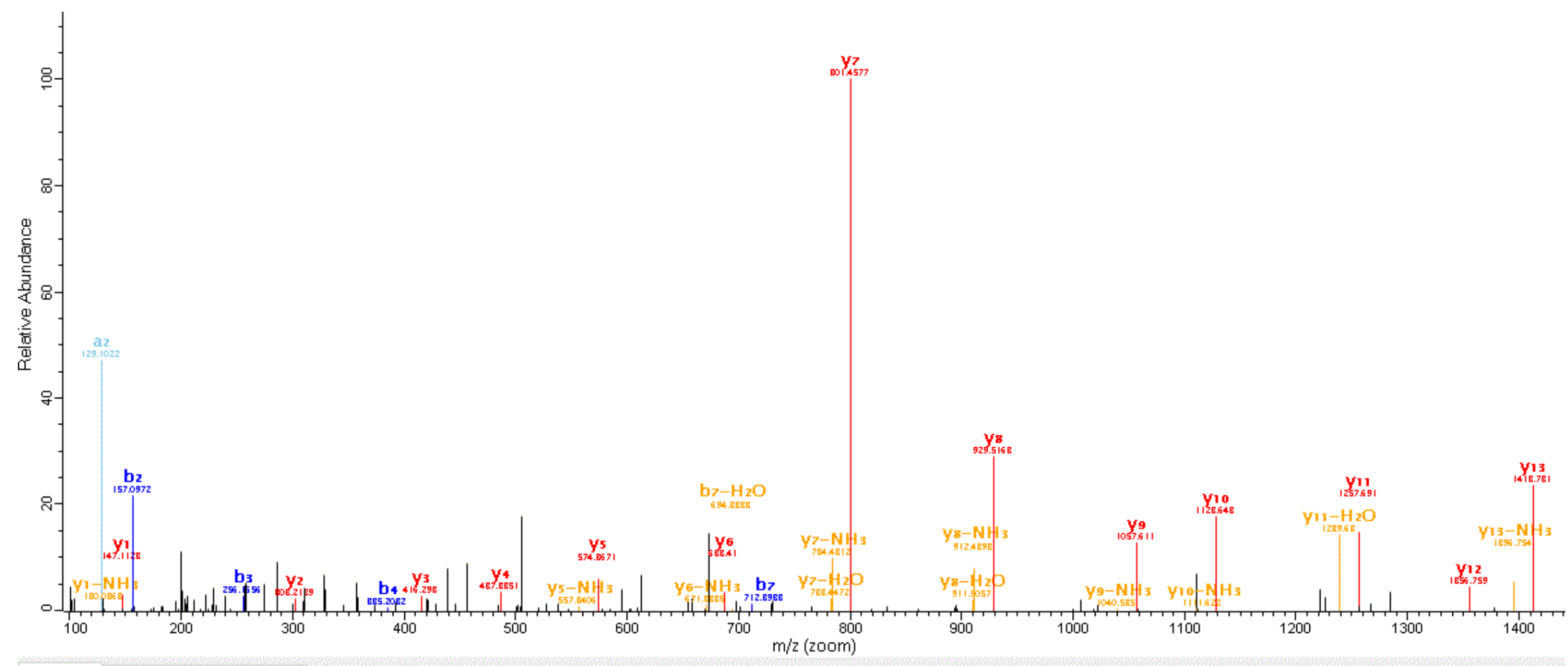

Sequence PSMs Fragments Properties

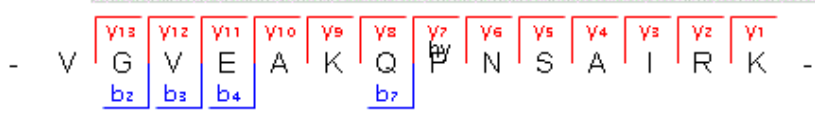

Figure S38. VGVEAKQP[Hydroxylation on P]NSAIRK. 